\title{
Multi-Depot Pickup and Delivery Problem with Resource Sharing
}

\author{
Yong Wang $\mathbb{D}^{1},{ }^{1}$ Lingyu Ran $\mathbb{D}^{1},{ }^{1}$ Xiangyang Guan $\mathbb{D}^{\circ},{ }^{2}$ and Yajie Zou $\mathbb{D}^{3}$ \\ ${ }^{1}$ School of Economics and Management, Chongqing Jiaotong University, Chongqing 400074, China \\ ${ }^{2}$ Department of Civil and Environmental Engineering, University of Washington, Seattle, WA 98195, USA \\ ${ }^{3}$ Key Laboratory of Road and Traffic Engineering of Ministry of Education, Tongji University, Shanghai 201804, China \\ Correspondence should be addressed to Yajie Zou; zouyajie@tongji.edu.cn
}

Received 10 April 2021; Revised 28 April 2021; Accepted 15 May 2021; Published 2 June 2021

Academic Editor: Xiaoyue Liu

Copyright $\odot 2021$ Yong Wang et al. This is an open access article distributed under the Creative Commons Attribution License, which permits unrestricted use, distribution, and reproduction in any medium, provided the original work is properly cited.

\begin{abstract}
Resource sharing (RS) integrated into the optimization of multi-depot pickup and delivery problem (MDPDP) can greatly reduce the logistics operating cost and required transportation resources by reconfiguring the logistics network. This study formulates and solves an MDPDP with RS (MDPDPRS). First, a bi-objective mathematical programming model that minimizes the logistics cost and the number of vehicles is constructed, in which vehicles are allowed to be used multiple times by one or multiple logistics facilities. Second, a two-stage hybrid algorithm composed of a $k$-means clustering algorithm, a Clark-Wright (CW) algorithm, and a nondominated sorting genetic algorithm II (NSGA-II) is designed. The $k$-means algorithm is adopted in the first stage to reallocate customers to logistics facilities according to the Manhattan distance between them, by which the computational complexity of solving the MDPDPRS is reduced. In the second stage, CW and NSGA-II are adopted jointly to optimize the vehicle routes and find the Pareto optimal solutions. CW algorithm is used to select the initial solution, which can increase the speed of finding the optimal solution during NSGA-II. Fast nondominated sorting operator and elite strategy selection operator are utilized to maintain the diversity of solutions in NSGA-II. Third, benchmark tests are conducted to verify the performance and effectiveness of the proposed two-stage hybrid algorithm, and numerical results prove that the proposed methodology outperforms the standard NSGA-II and multi-objective particle swarm optimization algorithm. Finally, optimization results of a real-world logistics network from Chongqing confirm the applicability of the mathematical model and the designed solution algorithm. Solving the MDPDPRS provides a management tool for logistics enterprises to improve resource configuration and optimize logistics operation efficiency.
\end{abstract}

\section{Introduction}

With the advancement of information technology and Internet of Things, the logistics industry is playing an increasingly important role in the development of modern businesses $[1,2]$. However, national and local governments worldwide are focusing on the environmental impacts of logistics and the efficient use of resources [3, 4]. In a logistics network, customers send out a series of requests for delivery and pickup services, and logistics service providers (LSPs) design service plans and arrange vehicles for these requests to deliver or pickup goods [5,6]. Efficient logistics service plan can improve the operation efficiency of LSPs and resource utilization $[7,8]$. Therefore, making an effective logistics service plan with resource sharing (RS) is essential, which not only helps to reduce the operating cost for logistics facilities but also promotes the development of green logistics and provides better logistics services for consumers $[9,10]$.

In this study, a multi-depot pickup and delivery problem with RS (MDPDPRS) combines components from three subproblems: multi-depot vehicle routing problem (MDVRP) with pickups and deliveries (MDVRPPD), MDVRP with pickups and deliveries and time windows (MDVRPPDTW), and RS [11-13]. However, one of the difficult challenges in solving this problem is how to handle pickup and delivery activities among multiple depots through RS $[14,15]$. In the traditional MDVRPPD, each vehicle performs only one type of activity in the service route, which may be delivering or picking up goods [16-18]. 
In addition, the traditional MDVRPPDTW and single-depot vehicle routing problem with pickups and deliveries mostly only consider the optimization of logistics operational costs $[19,20]$. Therefore, MDPDPRS focuses on how to support and achieve the efficient utilization of transportation resources with RS strategy, and optimizes the logistics network.

With regard to $\mathrm{RS}$, it is often jointly adopted with collaboration or cooperation between LSPs to optimize the logistics networks with multiple depots [21, 22]. RS strategy supports the sharing of customer information and transport resources to improve the resource configuration among logistics facilities to optimize the logistics network [23, 24]. Here, the sharing of customer information is often enabled by customer clustering, whereas the sharing of transportation resources is related to the use of shared transportation equipment $[25,26]$.

As for MDVRPPD, it is a crucial logistics issue with extensive applications, especially in reverse logistics [27]. Three basic types of vehicle routing problems exist in reverse logistics [28]. The first type is the vehicle routing problem with mixed deliveries and pickups, which involves customers with delivery demands, pickup demands, and delivery and pickup demands [29]. Vehicle routing problem with simultaneous delivery and pickup is the second type, which requires all customers to have both delivery and pickup demands [30]. The third type is the common MDVRPPD, which includes delivery and pickup customers in the logistics network [19,31-33]. In this study, the consideration of customer service time windows makes MDVRPPD realistic.

In this study, the MDPDPRS can be formulated into a biobjective mathematical model to minimize the total logistics operating cost and number of vehicles [32, 34]. On the basis of the multi-depot and RS properties of MDPDPRS, a two-stage hybrid algorithm is proposed to find the Pareto optimal solution. In the first stage, a $k$-means algorithm is adopted to reconfigure resources through customer clustering; thus, the MDVRPPDTW is simplified for solving [35]. The second stage focuses on finding the Pareto optimal solution for the bi-objective optimization problem $[23,36]$. The Clarke-Wright (CW) algorithm, which is good at constructing the initial solution of vehicle routes, and the nondominated sorting genetic algorithm (NSGA-II), which is known for its capability of finding the Pareto solution, are adopted to optimize the vehicle routing in the second stage $[33,37,38]$.

The remainder of this study is arranged as follows. Section 2 reviews the relevant literature. Section 3 elaborates the specifics of the MDPDPRS. Section 4 explains the bi-objective mathematical model for the MDPDPRS. Section 5 presents the designed methodology for solving the MDPDPRS. Section 6 analyzes the performance and application of the proposed model formulation and solution algorithm in a real-world case study compatible to the MDPDPRS. Finally, Section 7 summarizes the conclusions and discusses potential future research.

\section{Literature Review}

MDPDPRS is mainly related to the MDVRPPD, MDVRPPDTW, and RS strategy $[18,36]$. MDVRPPD and
MDVRPPDTW are the extension problems of MDVRP and MDVRPTW with respect to the logistics service type of customer demands, responsively [39, 40]. In the widely studied MDVRP and MDVRPTW, the service types of customers are either deliveries or pickups [16, 33, 41]. However, in a real-world logistics network with multiple depots, customers with distribution and pickup demands often exist simultaneously and the service time windows are the additional characteristics of customers; this issue is abbreviated by scholars as MDVRPPD [11, 13]. Therefore, MDVRPPD and MDVRPPDTW have begun to attract the attention of scholars, and the difference between the two issues is mainly whether customers' service time window feature is considered [42, 43]. In the MDVRPPDTW, the optimization of vehicle routes focuses on the confirmation of customers' service time windows and the integration of vehicles' delivery and pickup activities, which are suitable with the factors that LSPs must consider if optimizing vehicle routes [44, 45]. The adoption of RS into the optimization of logistics network is also a current trend [12]. Thus, the MDPDPRS in this study integrates vehicles' distribution and pickup arrangement with the RS strategy to optimize the MDVRPPDW.

In contrast to the MDVRP, the MDVRPPD studies customers with delivery and pickup demands [35, 42]. The MDVRPPDTW is an extension of MDVRPPD, which considers the characteristics of customer service time windows [46, 47]. Many scholars have studied MDVRPPD and MDVRPPDTW considering diverse aspects and proposed different mathematical models and algorithms $[30,48]$. In terms of models, many of the proposed mathematical models reflect the characteristics of their problem studied by different constraints, including capacity, time windows, and priority constraints [29, 49]. Ropke et al.[50] established a standard three-index model based on the characteristics of customer time window and designed an accurate algorithm to solve it. Gribkovskaia et al.[19] studied the vehicle routing problem with deliveries and pickups considering the number of times a customer has been visited and proposed a mixed integer linear programming model. Chen et al.[31] established a comprehensive mathematical model to minimize the transportation costs for unpaired vehicle routing problem with deliveries and pickups in a multi-factory production network. Conversely, hybrid heuristics algorithms (e.g., genetic algorithm and adaptive large neighborhood search algorithm) and exact algorithms based on column generation are commonly designed to solve MDVRPPD and MDVRPPDTW [50-52]. The proposed model and solution methodology in the above studies provide abundant reference for solving the basic MDVRPPD and MDVRPPDTW. However, few studies have optimized MDVRPPDTW with RS strategy $[32,34]$.

Customer clustering analysis is a research aspect that groups customers based on their characteristics (e.g., location and time window), and common clustering algorithms include $k$-means clustering, parallel clustering, and fuzzybased customer clustering [53-56]. In comparison with other customer clustering algorithms, $k$-means clustering is widely adopted to solve vehicle routing problems $[57,58]$. 
$\mathrm{Xu}$ et al.[59] proposed an enhanced ant colony algorithm based on $k$-means clustering to solve dynamic vehicle routing problems and achieved good optimization results. Hakim et al.[60] designed a cluster-based method to solve a vehicle routing problem with limited vehicle capacity, and their calculation results proved the effectiveness of that method. Mourelo Ferrandez et al.[61] reduced the calculation difficulty of truck-drone in tandem delivery network by $k$-means clustering algorithm. Wang et al.[35] proposed a hybrid heuristic algorithm based on three-dimensional $k$ means clustering and improved reference point NSGA-II to solve the multi-objective optimization model. Therefore, cluster analysis can simplify the difficulty of solving vehicle routing problem $[62,63]$.

At present, the construction of multi-objective optimization model and multi-objective optimization algorithm is the research hotspot of finding the Pareto solution of vehicle routing problems, and scholars have designed different algorithms [38, 64]. NSGA-II and multi-objective particle swarm optimization (MOPSO) algorithm are two common multi-objective algorithms $[65,66]$. NSGA-II adopts a reference point strategy to maintain population diversity [67]. Srivastava et al. [68] proposed a NSGA-II to solve the multiobjective optimization model for MDVRPTW and verified that the method is superior to the latest method of that problem through a real-world case study. Maadanpour Safari et al.[69] adopted NSGA-II, multi-objective simulated annealing (MOSA), and MOPSO to optimize the proposed three-objective mathematical function and concluded that NSGA-II was superior to MOSA according to the results of their examples. Shafiei Nikabadi et al. [70] formed a multiobjective model for the route selection of freight fleet, optimized it with NSGA-II and MOPSO, and considered that MOPSO was superior to NSGA-II. Therefore, NSGA-II and MOPSO are two commonly typical multi-objective optimization algorithms that can find Pareto solutions [71].

Many scholars have adopted the cooperation and RS strategy to optimize the multicenter logistics network $[11,17,24]$. Zhang et al. [15] believed that in the collaborative e-commerce truck carrier, participants can share transportation resources and customer demands to maximize the total profit of the whole alliance and improve vehicle utilization. Wang et al. [14] adopted the cooperation strategy to optimize the MDVRPPDTW with minimization of the operating cost of the transportation network and the total number of vehicles. Deng et al. [21] allowed the capabilities of the logistics facility, the vehicle resources, and the customer information to be shared through RS strategies, and they proved that this strategy can improve the utilization of logistics resources. In the study of Nourinejad et al. [72], vehicles can be used multiple times to reduce fleet size by extending vehicle reservation time. Li et al. [73] adopted the resource-sharing strategy to significantly optimize the logistics network and maximized the utilization of resources. Therefore, the adoption of RS strategy not only helps optimize the logistics costs but also improves the utilization of resources to protect the environment [22,74].

In summary, the existing literature has provided rich reference materials about MDVRPPD, MDVRPPDTW, and
RS, including model formulations and solution algorithms. However, the existing literature related to MDPDPRS has the following limitations. (1) Few studies on MDPDPRS have considered RS, MDVRPPD, and MDVRPPDTW. (2) The fact that a vehicle can be used multiple times on a working day is insufficiently considered in the proposed mathematical models. (3) Most of the proposed solution algorithms in the existing literature only address how to solve MDVRP, MDVRPPD, and MDVRPPDTW, and RS has not been incorporated into the designed algorithms. (4) Most of the existing literature focuses on raising problems and designing algorithms but neglects testing the proposed methods with practical cases.

In consideration of the aforementioned shortcomings, the main contributions of this study to MDPDPRS are as follows: (1) Characteristics of RS, MDVRPPD, and MDVRPPDTW are comprehensively incorporated to enrich the research on MDPDPRS. (2) On the basis of RS, this study proposes and tests that vehicles can be used multiple times and that customer information can be shared to save the transportation resources of logistics networks, which are considered in the proposed mathematical model. (3) A twostage algorithm is designed to combine RS with vehicle routing optimization to optimize the logistics network. (4) Benchmark and real-world cases are utilized to test and verify the performance and applicability of the proposed model and solution algorithm in this study.

\section{Problem Statement}

RS is an effective strategy that can optimize logistics operation costs and resource allocation in a multicenter logistics network with pickups and deliveries [21,35]. In this study, the logistics network consists of multiple distribution centers (DCs), multiple pickup centers (PCs), and multiple customers. The logistics network before and after optimization with RS, which is composed of DC1, DC2, DC3, PC1, PC2, and 52 customers (marked C1, C2, .., C52), is shown in Figure 1. The numerical number near the line represents the time distance between two elements (including facilities and customers).

In Figure 1(a), the unreasonable arrangement of vehicle routes and the nonsharing of resources are the main reasons for the higher operating costs of the logistics network. First, staggered driving and long-distance service are the two most significant unreasonable arrangements, and they cause additional travel costs. Second, vehicles that violate customers' time windows often occur. Arriving early and arriving late are generating penalty costs. Finally, the nonsharing of resources between facilities results in the capacity of facilities and transportation resources being left unused. For example, the vehicle no longer works after returning from $\mathrm{C} 2$ to $\mathrm{DC} 1$ in the service route $\mathrm{DC} 1 \longrightarrow \mathrm{C} 25 \longrightarrow \mathrm{C} 6 \longrightarrow \mathrm{C} 1 \longrightarrow \mathrm{C} 2$ $\longrightarrow$ DC1.

In Figure 1(b), the logistics network is optimized through the sharing of customers and transportation resources among facilities. First, the reallocation of customers between facilities facilitates the rearrangement of vehicles, which can avoid staggering and long-distance service. 

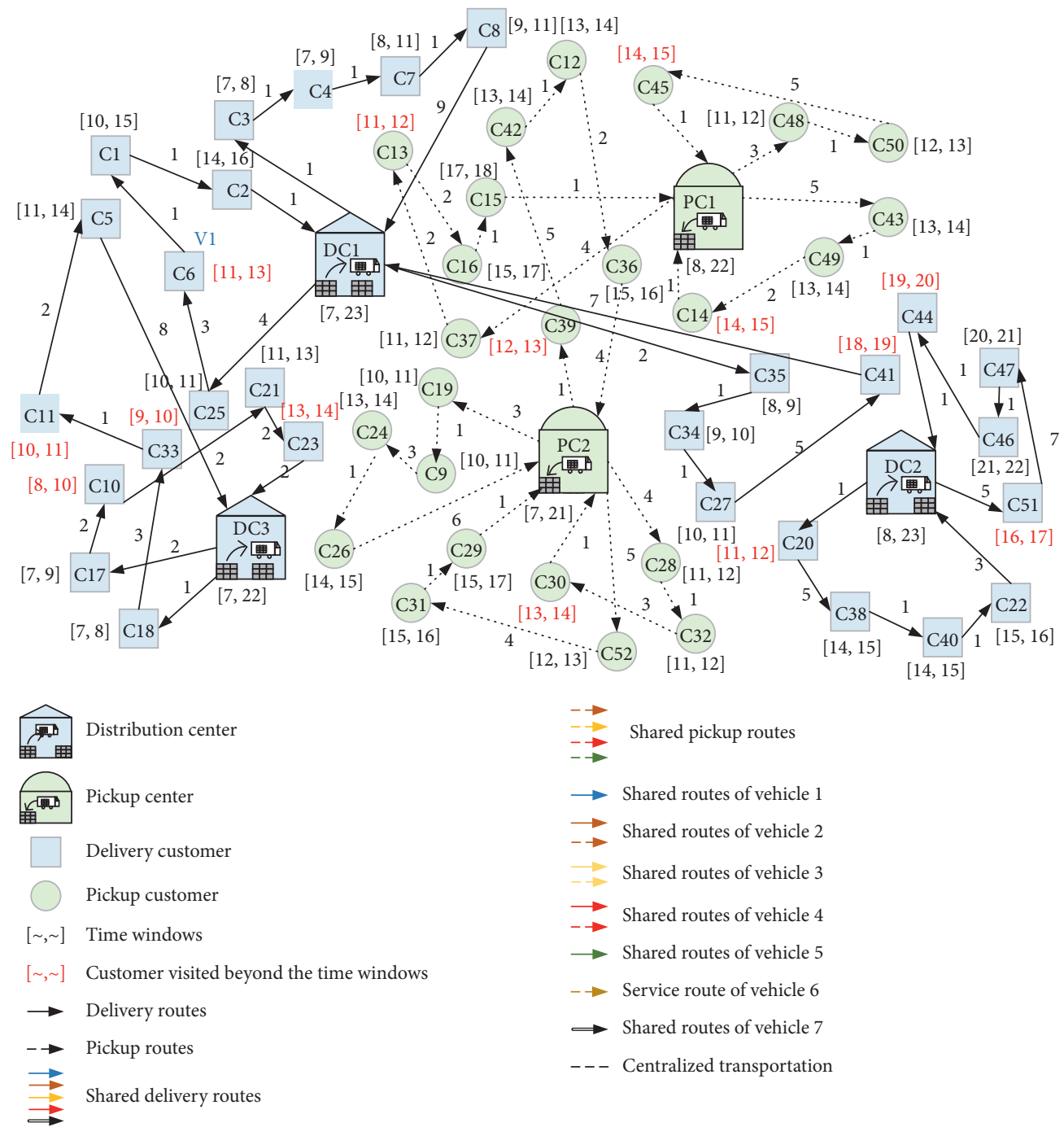

(a)

FIgUre 1: Continued. 


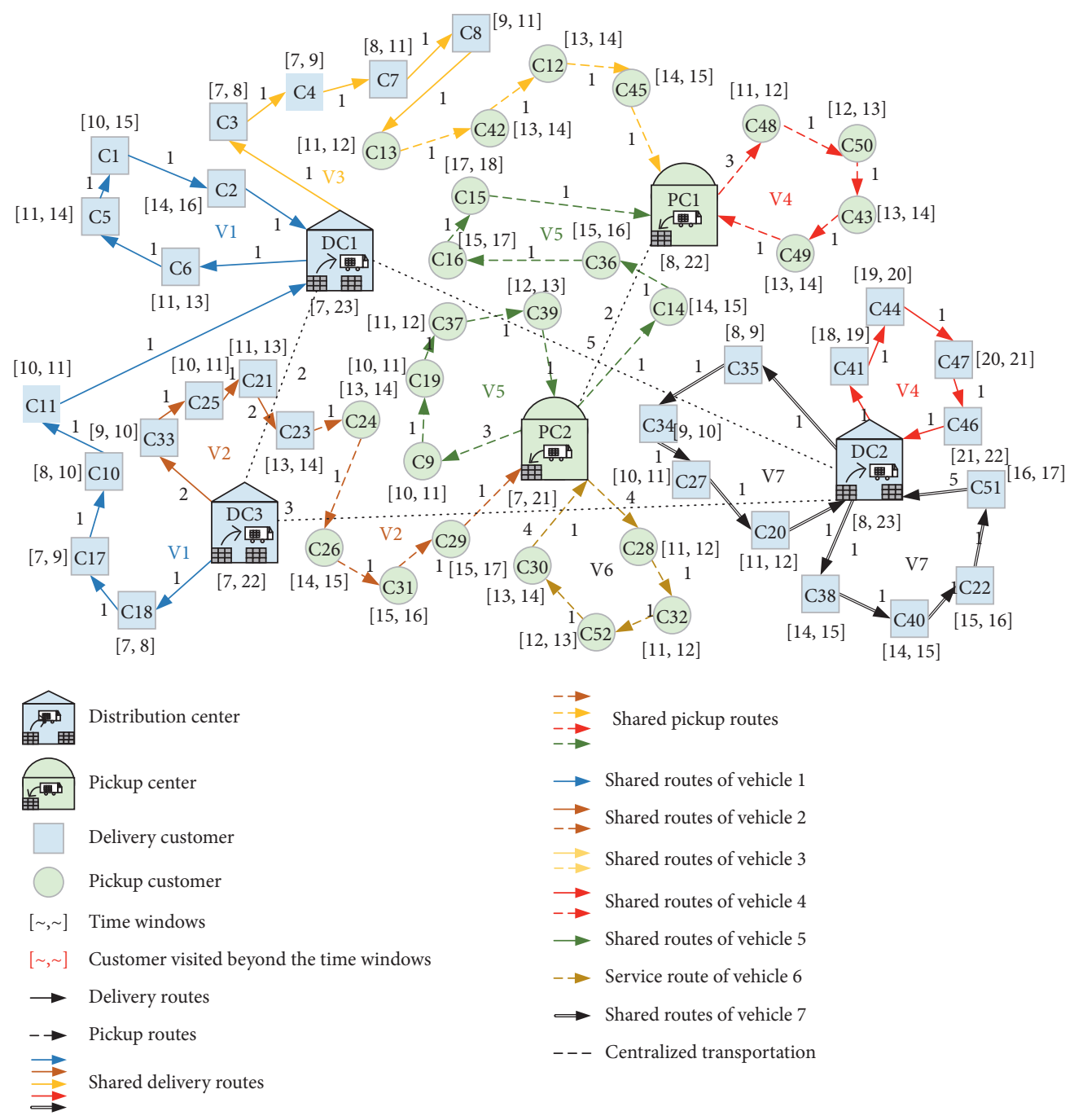

(b)

FIgURE 1: Logistics network optimization diagram of MDPDPRS. (a) Initial logistics network before resource sharing. (b) Optimized logistics network with resource sharing.

Second, vehicle resources can be better configured through sharing among multiple facilities. For example, $\mathrm{V} 1$ is used by DC3 and DC1. V2 and V3 have also implemented distribution and pickup services. The repeated use of vehicles prevents resources from being idle. Finally, the situation of vehicle violating the customers' time window is avoided.

To clearly demonstrate the discounts that RS brings to logistics network optimization, six indicators are counted, namely, total travel cost (TTC), total penalty cost (TPC), total maintenance cost (TMC), total fixed cost (TFC), number of vehicles (NV), and total operating cost (TOC), which are listed in Table 1. The unit time travel cost using a vehicle can be set to $\$ 10 / \mathrm{h}$, and the unit time penalty cost for waiting or late can be set to $\$ 20 / \mathrm{h}$, and the maintenance cost using a vehicle in a working period can be defined as $\$ 100$, and the fixed cost of a facility being used in a working period can be set to $\$ 200$.

In Table 1, the gap of TOC and NV in the initial and optimized logistics network is $\$ 1880$ and 7, respectively, which are highly significant. Before optimization, TTC (\$1500), TPC (\$440), and TMC (\$1400) are the main elements that cause additional logistics costs. In the optimized logistics network, the penalty costs are avoided, and the reduction in the number of vehicles also reduces the maintenance costs with RS. In addition, the travel costs of vehicles are also greatly reduced. Therefore, RS is an effective strategy for optimizing the logistics network of the MDPDPRS.

\section{Mathematical Model for MDPDPRS}

4.1. Assumptions and Notations. Some necessary assumptions can ensure the usability of the mathematical model [75] and those assumptions are listed as follows $[14,30,36,42,49,50]$.

Assumption 1. In a working period, the demand and coordination of customers are stable and known. 
Assumption 2. Centralized transportation among logistics facilities is performed by trucks.

Assumption 3. The vehicles can be used multiple times by the same or different logistics facilities.

The relevant notations and their descriptions are listed in Table 2.

4.2. Mathematical Model. A bi-objective mathematical model that minimizes the logistics operational costs (Equation (1)) and the number of vehicles (Equation (2)) is formed to solve the MDPDPRS. In Equation (1), the logistics operation cost is composed of four parts, which are marked by the TTC (Equation (3)), TPC (Equation (4)), TMC (Equation (5)), and TFC (Equation (6)). In Equation (2), $\sum_{c \in S_{F}} \sum_{h \in C_{U}} x_{v c h}$ represents the shared times of vehicle $v$.

$$
\begin{aligned}
\text { Min TOC }= & \text { TTC }+ \text { TPC }+ \text { TMC }+ \text { TFC } \\
\text { Min TNV }= & \sum_{v \in V} \min \left\{\sum_{c \in S_{F}} \sum_{h \in C_{U}} x_{v c h}, 1\right\} \\
\text { TTC }= & T \times \sum_{v \in V} \sum_{c \in C_{U} \cup S_{F}} \sum_{h \in C_{U} \cup S_{F}} x_{v c h} \times d_{c h} \times f_{v} \times P_{v} \\
& +T \times \sum_{b \in B} \sum_{o \in S_{F}} \sum_{w \in S_{F}} \sum_{f \in S_{F}} x_{b o w f} \times d_{w f} \times f_{b} \times P_{b}
\end{aligned}
$$

$$
\begin{aligned}
\mathrm{TPC}= & T \times \sum_{v \in V} \sum_{c \in C_{U}} \sum_{f \in S_{F}} \sum_{k \in R_{v}} x_{v c f k} \\
& \times\left(P_{E} \times \max \left\{E_{c}-A_{v c k}, 0\right\}+P_{L} \times \max \left\{A_{v c k}-L_{c}, 0\right\}\right),
\end{aligned}
$$

$\mathrm{TMC}=\sum_{v \in V} \frac{M_{v}}{W} \min \left\{R_{v}, 1\right\}+\sum_{b \in B} \sum_{o \in S_{F}} \sum_{w=o} \sum_{f \in S_{F}} \frac{M_{b}}{W} \times x_{b o w f}$,

$$
\mathrm{TFC}=\sum_{f \in S_{F}} \frac{z_{f} \times I_{f}}{W}
$$

Subject to

$$
\begin{aligned}
\sum_{v \in V} \sum_{f \in S_{F}} \sum_{k \in R_{v}} x_{v c f k} & =1, \quad \forall c \in C_{U}, \\
R_{v} & =\sum_{c \in S_{F}} \sum_{h \in C_{U}} x_{v c h}, \quad \forall v \in V, \\
\sum_{f \in S_{F}} x_{v c f k} & =1, \quad \forall v \in V, k \in R_{v}, c \in C_{U}, \\
\sum_{h \in C_{U}} x_{v c h}-\sum_{h \in C_{U}} x_{v h c} & =0, \quad \forall v \in V, c \in C_{U},
\end{aligned}
$$

$$
\sum_{c \in S_{F}} \sum_{h \in C_{U}} x_{v c h}=\sum_{h \in C_{U}} \sum_{h \in S_{F}} x_{v h n}, \quad \forall v \in V
$$

$\sum_{w=o, f \in S_{F}\{o\}} x_{\text {bowf }}=\sum_{w=o, f \in S_{F} \backslash\{o\}} x b o f w, \quad \forall b \in B, o \in S_{F}$,

$\sum_{w \in S_{F}} x b o w f-\sum_{w \in S_{F}} x_{b o f w}=0, \quad \forall b \in B, o, f \in S_{F}$,

$\sum_{c \in C_{D}} x_{v c f k} \times Q_{c}^{D} \leq C_{V}, \quad \forall v \in V, f \in S_{F}, k \in R_{v}$,

$\sum_{c \in C_{P}} x_{v c f k} \times Q_{c}^{P} \leq C_{V}, \quad \forall v \in V, f \in S_{F}, k \in R_{v}$,

$\sum_{f \in S_{F}} x_{b o f} \cdot Q_{o f} \leq C_{b}, \quad \forall b \in B, o \in S_{F}$

$$
\begin{aligned}
& Q_{o f}=\sum_{c \in C_{D}} x_{c o f} \times Q_{c}^{D}, \quad \forall o, f \in D, \\
& Q_{o f}=\sum_{c \in C_{P}} x_{c f o} \times Q_{c}^{P}, \quad \forall o, f \in P,
\end{aligned}
$$

$\sum_{v \in V} \sum_{c \in C_{D}} \sum_{k \in R_{v}} x_{v c f k} \times Q_{c}^{D} \leq C_{f}, \quad \forall f \in S_{D}$

$$
\sum_{v \in V} \sum_{c \in C_{P}} \sum_{k \in R_{v}} x_{v c f k} \times Q_{c}^{P} \leq C_{f}, \quad \forall f \in S_{P}
$$

$$
E_{f} \leq G_{v f k} \leq L_{f}, \quad \forall v \in V, c \in S_{F}, k \in R_{v}
$$

$$
A_{v c k}+t_{c f} \leq G_{v f(k+1)}, \quad \forall v \in V, c, f \in S_{F}, k \in R_{v}
$$

$$
E_{c} \leq A_{v c k} \leq L_{c}, \quad \forall v \in V, c \in S_{F} \cup C_{U}, k \in R_{v},
$$

$$
\begin{aligned}
& E_{h} \times x_{v c h} \leq\left(A_{v c k}+t_{c h}\right) \times x_{v c h} \leq L_{h} \times x_{v c h}, \\
& \forall v \in V, c \in C_{U}, h \in C_{U} \cup S_{F}, k \in R_{v},
\end{aligned}
$$

$$
\sum_{c, h \in S_{F} \cup C_{U}} x_{v c h} \times t_{c h} \leq T_{v}, \quad \forall v \in V
$$

$$
\begin{aligned}
\sum_{c, h \in C_{U}, r \neq h} x_{v c f k} \times x_{v c h} & =\sum_{c \in C_{U}} x_{v c f k}-1, \\
\forall v & \in V, f \in S_{F}, k \in R_{v},
\end{aligned}
$$

$\sum_{f, w \in S_{D} \backslash\{o\}} x_{b o w f} \leq \sum_{f \in S_{D} \backslash\{o\}} x_{b o f}-1, \quad \forall b \in B, o \in S_{D}$,

$\sum_{f, w \in S_{P} \backslash\{o\}} x_{b o w f} \leq \sum_{f \in S_{P} \backslash\{0\}} x_{b o f}-1, \quad \forall b \in B, o \in S_{P}$

$$
x_{v c f k}=\{0,1\}, \quad \forall v \in V, c \in C_{U}, f \in S_{F}, k \in R_{v},
$$


TABLE 1: Comparison of MDPDPRS before and after optimization.

\begin{tabular}{lcccccccc}
\hline & TTC (\$) & TPC $(\$)$ & TMC $(\$)$ & TFC $(\$)$ & NV & TOC (\$) & Gap \\
& & & 440 & 1400 & 1000 & 14 & 4340 & 7 \\
Initial & 1500 & 0 & 700 & 1000 & 7 & 2460 & 1880 \\
Optimized & 760 & 0 & TOC $(\$)$ \\
\hline
\end{tabular}

TABLE 2: Notations and description.

\begin{tabular}{|c|c|}
\hline Set & Description \\
\hline$S_{D}$ & Set of DCs, the total number of DCs is $N_{D}$ \\
\hline$S_{P}$ & Set of PCs, the total number of PCs is $N_{P}$ \\
\hline$S_{F}$ & Set of DCs and PCs, $S_{F}=S_{P} \cup S_{D}$ \\
\hline$C_{D}$ & Set of delivery customers \\
\hline$C_{P}$ & Set of pickup customers \\
\hline$C_{U}$ & Set of all customers, $U=C_{D} \cup C_{P}$ \\
\hline$V$ & Set of vehicles, the total number of vehicles is $N_{V}$ \\
\hline$B$ & Set of trucks, the total number of trucks is $N_{B}$ \\
\hline \multicolumn{2}{|c|}{ Parameters } \\
\hline$Q_{c}{ }^{D}$ & Delivery demand of customer $c, c \in C_{D}$ \\
\hline$Q_{c}{ }^{P}$ & Pickup demand of customer $c, c \in C_{P}$ \\
\hline$\left[E_{c} L_{c}\right]$ & Service time window of customer or logistics facility $c, c \in C_{U} \cup S_{F}$ \\
\hline$d_{c h}$ & Travel distance between customer or facility $c$ and customer $h . c, h \in C_{U} \cup S_{F}$ \\
\hline$t_{c h}$ & Travel time of a vehicle driving from customer or facility $c$ to customer $h . c, h \in C_{U} \cup S_{F}$ \\
\hline$P_{E}$ & Waiting penalty coefficient of arriving earliness (unit: dollars/unit time) \\
\hline$P_{L}$ & Tardiness penalty coefficient of arriving delay (unit: dollars/unit time) \\
\hline$W$ & Number of working periods in a year \\
\hline$T$ & Number of working days in a working period \\
\hline$T_{v}$ & Maximum travel time of vehicle $v, v \in V$ \\
\hline$T_{b}$ & Maximum travel time of truck $b, b \in B$ \\
\hline$f_{v}$ & Fuel consumption rate of vehicle $v$ per $\mathrm{km}$ (unit: gallon/miles) \\
\hline$f_{b}$ & Fuel consumption rate of truck $b$ per km (unit: gallon/miles) \\
\hline$P_{v}$ & Gasoline price (unit: dollars/gallon) \\
\hline$P_{b}$ & Gasoline price (unit: dollars/gallon) \\
\hline$C_{v}$ & Capacity of vehicle $v, v \in V$ \\
\hline$C_{b}$ & Capacity of truck $b, b \in B$ \\
\hline$C_{f}$ & Capacity of facility $f, f \in S_{F}$ \\
\hline$M_{v}$ & Annual maintenance cost of vehicle $v, v \in V$ \\
\hline$M_{b}$ & Annual maintenance cost of truck $b, b \in B$ \\
\hline$I_{f}$ & Annual fixed cost of facility $f, f \in S_{F}$ \\
\hline$R_{v}$ & The total service route number of vehicle $v, v \in V$ \\
\hline$G_{v f k}$ & The departure time of vehicle $v$ from facility $f$ in the $k$ th service route. $v \in V, f \in S_{F}, k \in R_{v}$ \\
\hline$A_{v c k}$ & The arriving time of vehicle $v$ at customer or facility $c$ in the $k$ th service route, $v \in V, c \in C_{U} \cup S_{F}, k \in R_{v}$ \\
\hline$Q_{o f}$ & The number of transported goods from facility $o$ to $f, o, f \in S_{F}$ \\
\hline \multicolumn{2}{|c|}{ Variables } \\
\hline$x_{v c f k}$ & $\begin{array}{l}x_{v c f k}=1 \text { if customer } c \text { is served by vehicle } v \text { departing from DC or PC } f \text { in the } k \text { th service route of vehicle } v \text {; otherwise, } x_{v c f k}=0 \text {, } \\
\qquad v \in V, c \in C_{U}, f \in S_{F}, k \in v\end{array}$ \\
\hline$x_{\text {cof }}$ & $x_{c o d}=1$ if the facility providing logistics service for customer $c$ is changed from facility $o$ to $f$; otherwise, $x_{c o d}=0, c \in C_{U}, o, f \in S_{F}$ \\
\hline$x_{v c h}$ & $x_{v c h}=1$ if vehicle $v$ travels directly from facility or customer $c$ to $h$; otherwise, $x_{v c h}=0, v \in V, c, h \in S_{F} \cup C_{U}$ \\
\hline$x_{\text {bof }}$ & $x_{b o f}=1$, if the goods transported from facility $o$ to $f$ is carried by truck $k$; otherwise, $x_{b o f}=0, b \in B, o, f \in S_{F}$ \\
\hline$x_{\text {bowf }}$ & $x_{\text {bowf }}=1$, if truck $k$ departs from facility $o$ and travels directly from facility $w$ to $f$; otherwise, $x_{\text {bowf }}=0, b \in B, o, w, f \in S_{F}$ \\
\hline$z_{f}$ & $z_{f}=1$ if facility $f$ agrees to share resource, otherwise, $z_{f}=0, f \in S_{F}$. \\
\hline
\end{tabular}

$$
\begin{array}{cl}
x_{c o f}=\{0,1\}, & \forall c \in C_{U}, o, f \in S_{F}, \\
x_{v c h}=\{0,1\}, & \forall v \in V, c, h \in C_{U} \cup S_{F}, \\
x_{b o f}=\{0,1\}, & \forall b \in B, o, f \in S_{F}, \\
x_{\text {bowf }}=\{0,1\}, \quad \forall b \in B, o, w, f \in S_{F},
\end{array}
$$

$$
z_{f}=\{0,1\}, \quad \forall f \in S_{f} .
$$

Constraint (7) ensures that each customer is served once. Constraint (8) counts the shared times of vehicle $v$. Constraints (9)-(11) ensure that flow conservation is achieved on each customer. Constraints (12) and (13) are the flow balance constraints of the truck. Constraints (14)-(16) ensure that the loading quantity of each vehicle and each truck cannot 
exceed their capabilities. Constraints (17) and (18) count the quantity of transshipment goods between logistics facilities. Constraints (19) and (20) guarantee that the total service quantity of each facility does not exceed its capacity. Constraints (21) and (22) require that the departure time and return time of each vehicle must meet the service time window of its served facility. Constraints (23) and (24) ensure that each vehicle must provide services for customers within the customers' service time window. Constraint (25) requires that the total working time of each vehicle does not exceed its maximum working time. Constraints (26)-(28) are used to eliminate the sub-tours of each vehicle and truck. The constraints of relevant binary variables are listed in Constraints (29)-(34).

\section{Solution Methodology for MDPDPRS}

MDVRPTW and MDVRPPDTW are typical NP-hard problems [12, 18, 41]. Multi-objective optimization algorithm and two-stage algorithm are often designed in combination to solve MDVRPPDTW [14, 23, 36]. Here, a two-stage algorithm with customer clustering first and then vehicle routing optimization is designed to solve MDPDPRS. This two-stage hybrid algorithm is composed of $k$-means, CW, and NSGA-II algorithms, and named KCW-NSGA-II. In the first stage of KCW-NSGAII, customers and resources are reconstructed by the $k$-means clustering algorithm $[57,58]$. The main purpose of the second stage is to optimize vehicle routes and find the Pareto optimal solution. The CW algorithm is adopted to construct the initial solution for NSGA-II [33, 37, 64, 65, 67].

The designed algorithm flow is illustrated in Figure 2. Here, Gen is the current number of iterations; MaxGen is the maximum number of iterations; $r$ is the number of iterations of the current internal re-optimization mechanism, which is between clustering and vehicle routing optimization; and $\operatorname{Max} R$ is its maximum number of iterations.

In Figure 2, the two-stage characteristics of customer clustering first and vehicle routing optimization later are clearly demonstrated. First, a $k$-means customer clustering mechanism based on Manhattan distance is designed. The clustering results are checked, updated, and saved after finishing the reallocation of all customers. Second, the CW algorithm is adopted to design the initial population and initial feasible solution, which accelerates the speed and possibility of NSGAII algorithm to find the Pareto optimal solution. Third, the elite strategy and genetic operation of NSGA-II are used to iteratively optimize the generated initial solution to find the Pareto optimal solution, which is mainly embodied in the change of Gen. Fourth, a regulatory re-optimization mechanism between customer clustering and vehicle routing optimization is set up to maintain gene stability during genetic operation, and this mechanism is implemented by the re-updating of $r$. Finally, if Gen is updated to MaxGen, then the iterative optimization of the algorithm is finished and the found Pareto optimal solution is outputted.

5.1. K-Means Clustering Algorithm. Customer clustering is an important measure to reduce the complexity of solving MDVRPPDTW [36]. $K$-means algorithm is widely used to solve MDVRPTW due to its simplicity and efficiency $[14,21]$. The $k$-means clustering pseudocode based on Manhattan distance is listed in Algorithm 1.

5.2. CW Algorithm. A common and effective way to construct the initial solution of VRP is the CW savings algorithm, which is actually a greedy heuristic algorithm $[33,37,76]$. The service time windows of customers and the capacity of vehicles are the main constraints to construct the initial solution $[77,78]$. The pseudocode of the CW algorithm designed in this study is listed in Algorithm 2.

5.3. NSGA-II. NSGA-II is a multi-objective optimization algorithm based on GA, which searches the Pareto optimal solution for multi-objective optimization [38, 64, 68]. Fast nondominated sorting operator, individual crowding distance operator, and elite strategy selection operator are the three key designs of NSGA-II $[33,65]$. Here, we suppose that the population is $P$ and $n$ individuals exist, and the individual objective function value of individual $i$ is $x_{i}$.

5.3.1. Fast Nondominated Sorting Operator. The key design of NSGA-II is to find the Pareto optimal solution. To enhance the possibility of finding the Pareto solution, the fast nondominated sorting operator stratifies the population $P$ according to the quality of individual solutions $[33,64,65,67]$. This method is a cyclic process of grading based on population fitness. Here, the nondominated solution set and the rank value assigned to the individual are the two indicators for fast nondominated sorting. Assume that $F_{i}$ represents the nondominated solution set, and $i_{\mathrm{r}}$ represents the rank value of individual $i$. Then, the pseudocode of the fast nondominated sort operator is shown in Algorithm 3.

5.3.2. Crowding Distance and Its Comparison Principle. The crowding distance is designed to drive the population to converge to the Pareto optimal solution and maintain the diversity of the population, which is mainly for individuals in the same nondominant layer $[38,64,68]$. We assume that $n$ individuals exist in the nondominant level of $S_{F}$, and the objective function value of individual $i$ is $x_{i}$. Then, the crowding distance is calculated as

$$
L(i)= \begin{cases}\infty, & i=1 \text { or } i=n, \\ \left|x_{i-1}-x_{i+1}\right|, & i=2,3, \ldots, n-1\end{cases}
$$

In Equation (35), $\left|x_{i-1}-x_{i+1}\right|$ represents the sum of the distance between individuals $i-1$ and $i+1$ in each direction of the objective function. Here, the objective function values of individuals need to be sorted before calculating the crowding distance. If the rank values of individuals $i$ and $j$ are $i_{\mathrm{r}}$ and $j_{\mathrm{r}}$, then the crowding distance is $L(i)$ and $L(j)$, respectively. The individual crowding comparison strategy based on crowding distance and nondominated ranking results is as follows. 


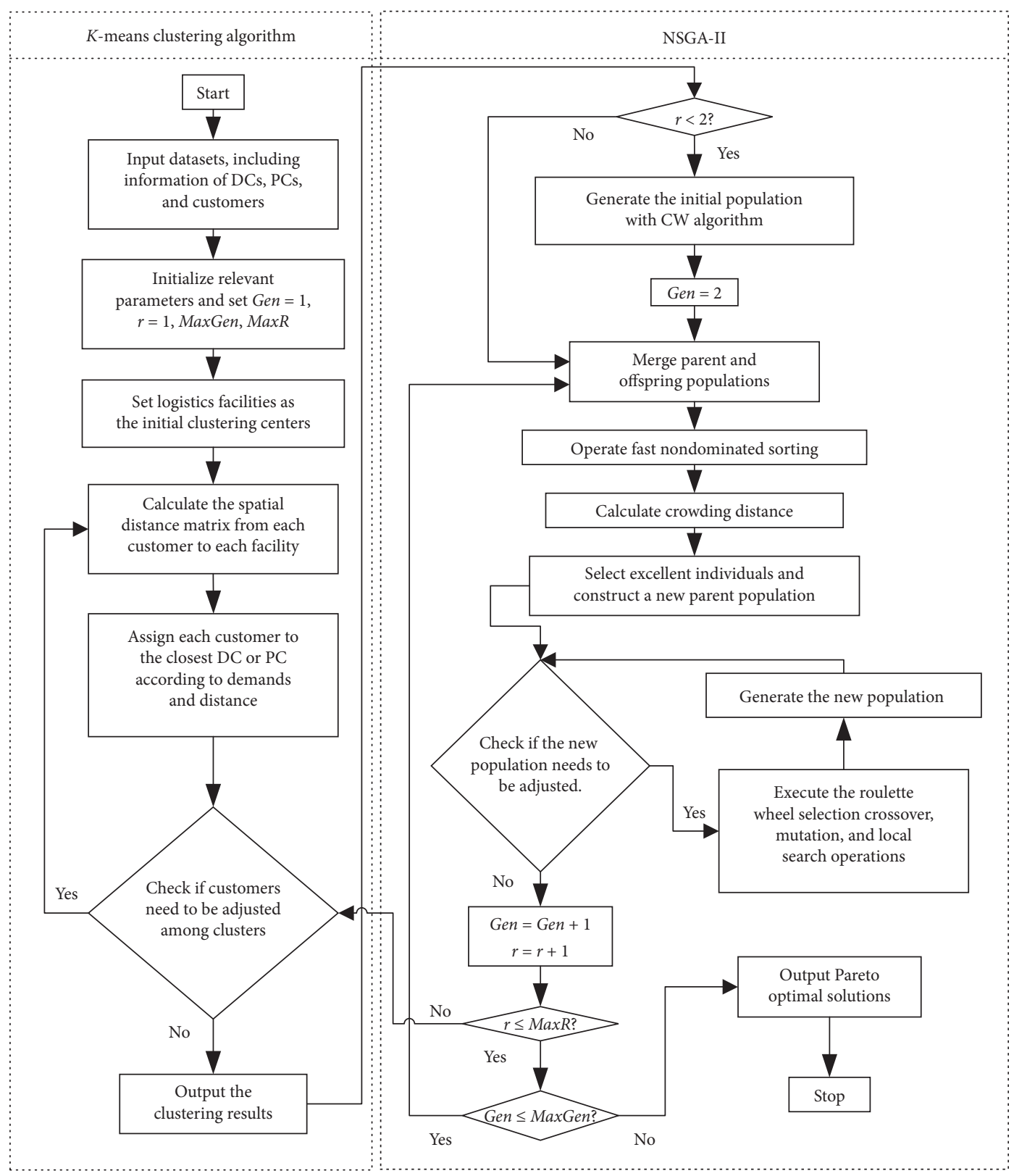

Figure 2: Algorithm flowchart of MDPDPRS.

Input: The datasets, including logistics facility and customer information, such as the coordination, time windows, and demands Output: The clustering results

(1) Step 1: Select $k$ objects as the initial clustering center

(2) Step 2: Calculate the Manhattan distance between each customer and each clustering center

(3) Step 3: (Re-)Assign each customer to their closest clustering center

(4) Step 4: If some customers need to be adjusted among the clustering results, then enter Step 3; otherwise, go to Step 5

(5) Step 5: Update the clustering centers

(6) Step 6: Output the clustering results

Algorithm 1: Procedure of $k$-means algorithm. 
(1) If $i_{\mathrm{r}}>j_{\mathrm{r}}$, then individual $i$ is the best one. If $i_{\mathrm{r}}<j_{\mathrm{r}}$, then individual $j$ is the best one.

(2) If $i_{\mathrm{r}}=j_{\mathrm{r}}$, then the individual with the most crowded distance is the better one.

5.3.3. Elite Strategy Selection Operator. To prevent the Pareto optimal solution from being lost in the iteration process, an elite strategy selection operator is designed, which selects the optimal solution by nondominated sorting and crowding distance between the parent and offspring populations. Suppose that the current iteration is $t$ and the parent population is $P_{t}$, the offspring population is $Q_{t}$, and $R_{t}$ is composed by $P_{t}$ and $Q_{t}$. First, a fast nondominated sorting is performed for $R_{t}$, and the crowding distance is then calculated. On the basis of the crowding distance and the nondominated layer, $N$ individuals with high-quality solution is selected to form a new population $P_{t+1}$.

\section{Empirical Analyses}

6.1. Algorithm Comparison. The standard NSGA-II, GA$\mathrm{PSO}$, and MOPSO are adopted for comparison to verify the applicability and effectiveness of the proposed KCW-NSGA-II algorithm in solving MDPDPRS [38, 64-66, 68, 79]. The benchmark dataset C-mdvrptw (consisting of instances of 20 groups) is utilized for the test, which is mainly obtained from the website. Networking and emerging optimization and their related characteristics are listed in Table 3. To meet the characteristics of the research object in this study, depots are regarded as logistics facilities, and customers are divided into two types, that is, those with distribution demands and those with pickup demands.

In Table 3, the number of customers and logistics facilities in each instance is different. The first instance comprises four facilities and 48 customers, whereas Instance 10 includes six facilities and 288 customers. In addition, the loading capacity of vehicle used in each instance is differentiated.

Relevant parameters are properly unified to mitigate their effects on algorithm performance. These parameters are set as follows [38, 64-66, 68, 79]: (1) parameters about GA: population size popsize $=200$, selection possibility $s p=0.6$, crossover possibility $c p=0.9$, mutation possibility $m p=0.2$; (2) parameters about PSO: popsize $=200$, inertia weight $i w=0.85$, the personal learning confidences $p c=2$, and social learning confidence $g c=3$; and (3) other relevant parameters: maximum number of generation genmax $=1200$ and velocity of vehicle $v=5$. The costs, number of vehicles $(\mathrm{NV})$, and computation time (CT) are calculated to verify the performance of the algorithms and the numerical results are listed in Table 4.

The numerical results shown in Table 4 demonstrate that the proposed algorithm KCW-NSGA-II performs better than the other three algorithms. First, the average cost of the four algorithms is $\$ 2584, \$ 2944, \$ 2746$, and $\$ 3025$, respectively. By contrast, the costs and the number of vehicles optimized by KCW-NSGA-II are the most economical solution compared with the other three algorithms in each instance. Second, the value of t-test also shows that the KCW-NSGA-II is significantly different from the other three algorithms. In addition, the proposed algorithm can obtain the optimal solution quickly. Therefore, the proposed algorithm KCW-NSGA-II outperforms the other three algorithms. Moreover, this algorithm can be adjusted to address problems such as VRPMDP, VRPSDP, and PVRP.

6.2. Data Source and Relevant Parameter Setting. As an inland international logistics hub and an open highland, Chongqing is a new first-tier city in China. Therefore, as our numerical experiments, the logistics network adopted from Chongqing is appropriate to verify the applicability of this study. Six logistics facilities (i.e., DC1, DC2, DC3, PC1, PC2, and PC3) and 220 customers are the main elements of this real-world logistics network. The information and characteristics of these elements are listed in Table 5, and the spatial allocation information is plotted in Figure 3.

In Table 5 , the number of customers served by the six logistics facilities is $27,36,39,46,31$, and 41 , respectively. In Figure 3, an obvious feature is that the customer allocation of each facility is relatively dispersed. The service area edge of each facility is not a clear division. In Table 6, the initial vehicle routes for the logistics network are shown, including the specific information of each service route.

In Table 6, the total number of vehicles used in the initial logistics network is 33 , and the number of vehicles used at each facility is $5,6,6,6,4$, and 6 , respectively. In addition, some vehicles return to their origin early, such as V9, V10, $\mathrm{V} 17$, and V18, indicating that these vehicle resources are underutilized. The service vehicle routes of DC1 and the 27 customers it serves in the logistics network are shown in Figure 4.

In Figure 4, the service routes of V3, V4, and V5 are relatively complex. V5 performs delivery services for customers C23, C22, C6, C16, C2, and C5. However, these customers may be closer to DC3 on the basis of the perspective of spatial distribution. Therefore, optimizing this logistics network is necessary. The values of the relevant parameters used in this real-world case study are shown in Table $7[38,64,65,68]$.

6.3. Optimization Results. Clustering customers to optimize resource allocation is the first step in optimizing the logistics network. The customer clustering results of this logistics network by $k$-means algorithm are shown in Figure 5.

In Figure 5, the service relationship between customers and facilities is optimized by clustering. Each customer is covered by the logistics facility that is located close to that customer. On the whole, the service area of each logistics facility has been obviously allocated. For example, C23, C22, C6, C16, and $\mathrm{C} 2$ are served by $\mathrm{DC} 1$ before clustering; however, they are also served by DC3. Statistical analysis of customers whose service relationship has changed like those five customers is the key to handle centralized transportation. The details of the amount of goods transferred among facilities are shown in Figure 6. 
Input: The datasets of customer and facility information including location, time window, demand, and other relevant parameters Output: The initial feasible solution

(1) Step 1: Mark the initial distance saving values with numbers 1, 2, 3, ., (n-1)

(2) Step 2: Assign vehicles to each customer

(3) Step 3: Calculate the distance savings of new vehicle routes, which are formed by any two routes

(4) Step 4: Sort the distance savings in descending order

(5) Step 5: If new vehicle routes meet the time window and capacity constraints, then go directly to Step 6; otherwise set $\mathrm{n}=n+1$ and return to Step 5

(6) Step 6: Generate a new vehicle route with two vehicle routes whose distance savings are maximum

(7) Step 7: Update the distance savings value through the fusion of vehicle routes

(8) Step 8: Generate new vehicle route with two vehicle routes whose distance savings are maximum

(9) Step 9: $n=1$

(10) Step 10: If new vehicle routes meet the time window and capacity constraints, then go directly to Step 11; otherwise, return to Step 7

(11) Step 11: $n=n+1$

(12) Step 12: Generate new vehicle route with two vehicle routes whose distance savings are maximum

(13) Step 13: If there exists a route that serves only one, then go back to Step 7; otherwise enter Step 14

(14) Step 14: Output the service route of each vehicle

Algorithm 2: Procedure of CW algorithm.

Input: Initial population $P$

Output: The nondominated sorting results of population $P$

(1) Step 1: Setting $P P=P$, rank=1, $F=1$

(2) Step 2: for $F=1: n$

(3) Step 3: for $i=1: \mathrm{n}$

(4) Step 4: for $j=1:$ : \&\& $j \neq i$

(5) Step 5: Compare the solution quality of $x_{i}$ and $x_{j}$, and determine the dominant and nondominant relationships of $i$ and $j$. End

(6) Step 6: If $x_{i}$ is superior to all $x_{j}$, then the individual $i$ is considered to be a nondominant individual, and the nondominant ranking value of $i$ is rank

End

(7) Step 7: The nondominant individuals found in the above steps constitute set $S_{F}$, which is regarded as the $F$-level nondominant layer of the population $P$

(8) Step 8: $P P=P P \backslash S_{F}, i_{\mathrm{r}}=i_{\mathrm{r}}+1$

(9) Step 9: If $P P$ is an empty set, then enter Step 10, otherwise continuous this cycle

(10) Step 10: Stratify population $P$

(11) Step 11: End

Algorithm 3: Procedure of the fast nondominated sorting operator.

In Figure 6, the amount of goods shipped from DC1 to DC2 is zero; thus, the initial customers of DC1 are not allocated to DC2 with clustering. The amount of goods transported from DC2 to DC1 is 170 , which indicates that the service relationship of some customers has changed from DC2 to DC1. Here, the spatial distance between DC1 and DC2 is $33.07 \mathrm{~km}$. The centralized transportation service routes of the truck are listed in Table 8 .

In Table 8, T1 and T2 serve three routes. At the end of each service route, the trucks should return to their origin. The activity of T1 occurs early on each workday, and that of T2 occurs late on each workday. Table 9 shows the optimized vehicle service routes.

In Table 9, the number of vehicles used jointly by the six logistics facilities is 12 . Some of the vehicles are used multiple times within and between the facilities. For example, V1 performs the route $\mathrm{DC} 1 \longrightarrow \mathrm{C} 86 \longrightarrow \mathrm{C} 13$ $\longrightarrow \mathrm{C} 20 \longrightarrow \mathrm{C} 11 \longrightarrow \mathrm{C} 4 \longrightarrow \mathrm{C} 12 \longrightarrow \mathrm{C} 7 \longrightarrow \mathrm{C} 5 \longrightarrow \mathrm{C} 1$ $\longrightarrow \mathrm{C} 25 \longrightarrow \mathrm{C} 3 \longrightarrow \mathrm{C} 8 \longrightarrow \mathrm{DC} 1$ and the route $\mathrm{DC} 3 \longrightarrow$ $\mathrm{C} 73 \longrightarrow \mathrm{C} 97 \longrightarrow \mathrm{C} 42 \longrightarrow \mathrm{C} 2 \longrightarrow \mathrm{C} 82 \longrightarrow \mathrm{C} 29 \longrightarrow \mathrm{C} 16$ $\longrightarrow \mathrm{C} 40 \longrightarrow$ DC3, which occur in DC1 and DC3, respectively. V10 provides service for PC2 and PC3 successively. V2, V4, and V6 are shared in DC1, DC2, and DC3, respectively.

To clarify the effect of the proposed model and algorithm, the gap of the cost and the number of vehicles in the initial and optimized logistics network are counted and listed in Table 10. Here, the TOC of the logistics facility includes the TTC, TFC, TPC, and TMC. Centralized transportation is a special project generated by the sharing of customer information and transportation resources among facilities. Therefore, the costs of centralized transportation should be jointly borne by all the facilities participating in the sharing. Similarly, given that 
TABLE 3: Characteristics of benchmark datasets.

\begin{tabular}{|c|c|c|c|c|c|c|}
\hline \multirow[t]{2}{*}{ Instance } & \multirow[t]{2}{*}{ Datasets } & \multirow[t]{2}{*}{ Maximum loading capacity } & \multicolumn{2}{|c|}{$\begin{array}{c}\text { Number of } \\
\text { depots }\end{array}$} & \multicolumn{2}{|c|}{ Number of customers } \\
\hline & & & DCs & PCs & Delivery demands & Pickup demands \\
\hline 1 & C-mdvrptw-pr01 & 200 & 2 & 2 & 24 & 24 \\
\hline 2 & C-mdvrptw-pr02 & 195 & 2 & 2 & 48 & 48 \\
\hline 3 & C-mdvrptw-pr03 & 190 & 2 & 2 & 72 & 72 \\
\hline 4 & C-mdvrptw-pr04 & 185 & 2 & 2 & 96 & 96 \\
\hline 5 & C-mdvrptw-pr05 & 180 & 2 & 2 & 120 & 120 \\
\hline 6 & C-mdvrptw-pr06 & 175 & 2 & 2 & 144 & 144 \\
\hline 7 & C-mdvrptw-pr07 & 200 & 3 & 3 & 36 & 36 \\
\hline 8 & C-mdvrptw-pr08 & 190 & 3 & 3 & 72 & 72 \\
\hline 9 & C-mdvrptw-pr09 & 180 & 3 & 3 & 108 & 108 \\
\hline 10 & C-mdvrptw-pr10 & 170 & 3 & 3 & 144 & 144 \\
\hline 11 & C-mdvrptw-pr11 & 200 & 2 & 2 & 24 & 24 \\
\hline 12 & C-mdvrptw-pr12 & 195 & 2 & 2 & 48 & 48 \\
\hline 13 & C-mdvrptw-pr13 & 190 & 2 & 2 & 72 & 72 \\
\hline 14 & C-mdvrptw-pr14 & 185 & 2 & 2 & 96 & 96 \\
\hline 15 & C-mdvrptw-pr15 & 180 & 2 & 2 & 120 & 120 \\
\hline 16 & C-mdvrptw-pr16 & 175 & 2 & 2 & 144 & 144 \\
\hline 17 & C-mdvrptw-pr17 & 200 & 3 & 3 & 36 & 36 \\
\hline 18 & C-mdvrptw-pr18 & 190 & 3 & 3 & 72 & 72 \\
\hline 19 & C-mdvrptw-pr19 & 180 & 3 & 3 & 108 & 108 \\
\hline 20 & C-mdvrptw-pr20 & 170 & 3 & 3 & 144 & 144 \\
\hline
\end{tabular}

TABLE 4: Comparison results of the four algorithms.

\begin{tabular}{|c|c|c|c|c|c|c|c|c|c|c|c|c|}
\hline \multirow{2}{*}{ Instance } & \multicolumn{3}{|c|}{ KCW-NSGA-II } & \multicolumn{3}{|c|}{ NSGA-II } & \multicolumn{3}{|c|}{ GA-PSO } & \multicolumn{3}{|c|}{ MOPSO } \\
\hline & Cost & $\mathrm{NV}$ & $\mathrm{CT}$ & Cost & NV & $\mathrm{CT}$ & Cost & NV & $\mathrm{CT}$ & Cost & NV & $\mathrm{CT}$ \\
\hline 1 & 1033 & 4 & 71 & 1178 & 4 & 77 & 1298 & 5 & 82 & 1593 & 5 & 98 \\
\hline 2 & 1787 & 8 & 72 & 1724 & 9 & 91 & 2172 & 10 & 93 & 2115 & 8 & 119 \\
\hline 3 & 2742 & 17 & 110 & 3121 & 18 & 133 & 2879 & 18 & 103 & 3309 & 18 & 127 \\
\hline 4 & 3216 & 20 & 129 & 4027 & 21 & 146 & 3371 & 21 & 171 & 3579 & 21 & 184 \\
\hline 5 & 3693 & 18 & 181 & 3827 & 22 & 239 & 3835 & 22 & 217 & 4121 & 30 & 264 \\
\hline 6 & 3569 & 15 & 306 & 3671 & 23 & 290 & 4008 & 24 & 301 & 4577 & 33 & 298 \\
\hline 7 & 1687 & 7 & 85 & 2386 & 9 & 83 & 1767 & 8 & 97 & 2044 & 14 & 79 \\
\hline 8 & 2093 & 12 & 95 & 2394 & 13 & 141 & 2181 & 12 & 114 & 2361 & 12 & 147 \\
\hline 9 & 2614 & 19 & 218 & 4010 & 22 & 210 & 2653 & 20 & 203 & 3167 & 30 & 240 \\
\hline 10 & 3123 & 24 & 258 & 3355 & 24 & 311 & 3277 & 25 & 277 & 3459 & 29 & 325 \\
\hline 11 & 1118 & 4 & 77 & 1405 & 5 & 74 & 1190 & 6 & 85 & 1754 & 11 & 101 \\
\hline 12 & 2068 & 10 & 94 & 2692 & 11 & 96 & 2394 & 11 & 88 & 2135 & 11 & 114 \\
\hline 13 & 2570 & 18 & 97 & 2655 & 18 & 103 & 2690 & 22 & 119 & 3282 & 28 & 99 \\
\hline 14 & 3028 & 21 & 162 & 3564 & 24 & 186 & 3108 & 23 & 162 & 3620 & 25 & 172 \\
\hline 15 & 3709 & 22 & 209 & 4117 & 26 & 240 & 3812 & 27 & 241 & 4272 & 33 & 248 \\
\hline 16 & 3993 & 25 & 262 & 4072 & 25 & 305 & 4154 & 28 & 301 & 4366 & 34 & 339 \\
\hline 17 & 1578 & 7 & 69 & 1763 & 7 & 87 & 1665 & 7 & 65 & 1652 & 7 & 126 \\
\hline 18 & 2324 & 12 & 66 & 2428 & 12 & 110 & 2430 & 14 & 109 & 2635 & 19 & 166 \\
\hline 19 & 2440 & 20 & 212 & 2790 & 21 & 206 & 2493 & 22 & 202 & 3029 & 22 & 241 \\
\hline 20 & 3295 & 23 & 186 & 3692 & 25 & 316 & 3546 & 25 & 213 & 3421 & 26 & 309 \\
\hline Average & 2584 & 15 & 148 & 2944 & 17 & 172 & 2746 & 18 & 162 & 3025 & 21 & 190 \\
\hline$t$-test & & & & -13.95 & -3.73 & -3.29 & -6.44 & -4.76 & -3.41 & -8.60 & -4.84 & -5.31 \\
\hline p-value & & & & $9.8 \mathrm{E}-12$ & 7.2E-04 & $1.9 \mathrm{E}-03$ & $1.8 \mathrm{E}-06$ & $6.8 \mathrm{E}-05$ & $1.5 \mathrm{E}-03$ & $2.8 \mathrm{E}-08$ & 5.7E-05 & $2.0 \mathrm{E}-05$ \\
\hline
\end{tabular}

the vehicle is shared and some vehicles are used multiple times, the maintenance of the vehicles should be co-paid by all facilities.

In Table 10, the fixed costs of each facility are stable, which are $\$ 315, \$ 427, \$ 533, \$ 578, \$ 612$, and $\$ 590$, respectively. Reducing the travel, penalty, and maintenance costs is the main objective of optimizing the logistics operating cost. The TOC of the initial logistics network is $\$ 25473$, whereas the optimized TOC is $\$ 16614$, which indicates that the logistics network is significantly improved. Transportation resources are greatly saved, as the number of vehicles before and after optimization is 33 and 14, respectively. The gap of TTC before and after optimization is shown in Figure 7, which can directly prove the optimization effect of vehicle routes. 
TABle 5: Characteristics of the logistics network.

\begin{tabular}{llcc}
\hline Symbol & \multicolumn{1}{c}{ Description } & Number of served customers & Mark of customers \\
\hline & DC1 and its customers & 27 & C1 - C27 \\
& DC2 and its customers & 36 & C28 - C63 \\
& DC3 and its customers & 39 & C64 - C102 \\
& PC1 and its customers & 46 & C103-C148 \\
& PC2 and its customers & 31 & C149- C179 \\
\hline & PC3 and its customers & 41 & C180 - C220 \\
\hline
\end{tabular}

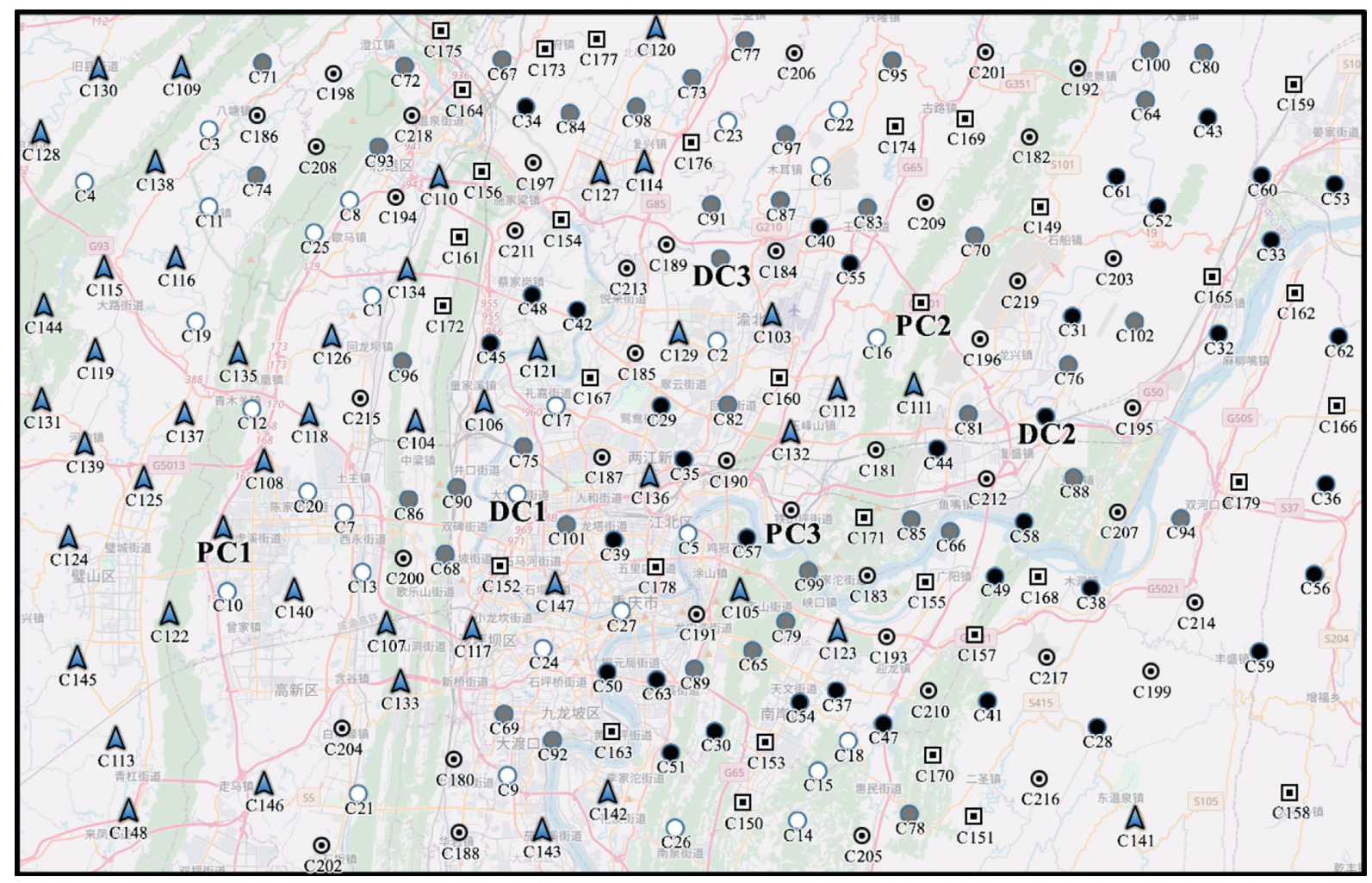

FIgURE 3: Spatial distribution of DCs, PCs, and their customers.

TABLE 6: Distribution routes of DCs and pickup routes of PCs in the initial logistics network.

\begin{tabular}{|c|c|c|c|c|}
\hline Facility & Vehicle & $\begin{array}{c}\text { Departure } \\
\text { time }\end{array}$ & $\begin{array}{c}\text { Arriving } \\
\text { time }\end{array}$ & Route \\
\hline \multirow{5}{*}{ DC1 } & V1 & 162 & 689 & $\mathrm{DC} 1 \longrightarrow \mathrm{C} 19 \longrightarrow \mathrm{C} 1 \longrightarrow \mathrm{C} 17 \longrightarrow \mathrm{DC} 1$ \\
\hline & $\mathrm{V} 2$ & 0 & 906 & $\mathrm{DC} 1 \longrightarrow \mathrm{C} 13 \longrightarrow \mathrm{C} 7 \longrightarrow \mathrm{C} 20 \longrightarrow \mathrm{C} 12 \longrightarrow \mathrm{DC} 1$ \\
\hline & $\mathrm{V} 3$ & 159 & 619 & $\mathrm{DC} 1 \longrightarrow \mathrm{C} 8 \longrightarrow \mathrm{C} 25 \longrightarrow \mathrm{C} 11 \longrightarrow \mathrm{C} 3 \longrightarrow \mathrm{C} 4 \longrightarrow \mathrm{C} 10 \longrightarrow \mathrm{DC} 1$ \\
\hline & $\mathrm{V} 4$ & 33 & 730 & $\mathrm{DC} 1 \longrightarrow \mathrm{C} 27 \longrightarrow \mathrm{C} 24 \longrightarrow \mathrm{C} 21 \longrightarrow \mathrm{C} 9 \longrightarrow \mathrm{C} 26 \longrightarrow \mathrm{C} 14 \longrightarrow \mathrm{C} 15 \longrightarrow \mathrm{C} 18 \longrightarrow \mathrm{DC} 1$ \\
\hline & V5 & 0 & 924 & $\mathrm{DC} 1 \longrightarrow \mathrm{C} 23 \longrightarrow \mathrm{C} 22 \longrightarrow \mathrm{C} 6 \longrightarrow \mathrm{C} 16 \longrightarrow \mathrm{C} 2 \longrightarrow \mathrm{C} 5 \longrightarrow \mathrm{DC} 1$ \\
\hline \multirow{6}{*}{ DC2 } & V6 & 0 & 1037 & $\mathrm{DC} 2 \longrightarrow \mathrm{C} 42 \longrightarrow \mathrm{C} 45 \longrightarrow \mathrm{C} 48 \longrightarrow \mathrm{C} 34 \longrightarrow \mathrm{C} 35 \longrightarrow \mathrm{C} 50 \longrightarrow \mathrm{C} 54 \longrightarrow \mathrm{DC} 2$ \\
\hline & V7 & 30 & 1016 & $\mathrm{DC} 2 \longrightarrow \mathrm{C} 58 \longrightarrow \mathrm{C} 39 \longrightarrow \mathrm{C} 41 \longrightarrow \mathrm{C} 47 \longrightarrow \mathrm{C} 37 \longrightarrow \mathrm{C} 30 \longrightarrow \mathrm{C} 43 \longrightarrow \mathrm{C} 40 \longrightarrow \mathrm{DC} 2$ \\
\hline & V8 & 0 & 1014 & $\mathrm{DC} 2 \longrightarrow \mathrm{C} 46 \longrightarrow \mathrm{C} 61 \longrightarrow \mathrm{C} 28 \longrightarrow \mathrm{C} 59 \longrightarrow \mathrm{C} 36 \longrightarrow \mathrm{C} 32 \longrightarrow \mathrm{C} 62 \longrightarrow \mathrm{C} 52 \longrightarrow \mathrm{C} 60 \longrightarrow \mathrm{DC} 2$ \\
\hline & V9 & 0 & 319 & $\mathrm{DC} 2 \longrightarrow \mathrm{C} 63 \longrightarrow \mathrm{C} 51 \longrightarrow \mathrm{C} 49 \longrightarrow \mathrm{DC} 2$ \\
\hline & V10 & 35 & 823 & $\mathrm{DC} 2 \longrightarrow \mathrm{C} 56 \longrightarrow \mathrm{C} 55 \longrightarrow \mathrm{C} 38 \longrightarrow \mathrm{C} 53 \longrightarrow \mathrm{DC} 2$ \\
\hline & V11 & 10 & 1196 & $\mathrm{DC} 2 \longrightarrow \mathrm{C} 31 \longrightarrow \mathrm{C} 33 \longrightarrow \mathrm{C} 44 \longrightarrow \mathrm{C} 29 \longrightarrow \mathrm{C} 57 \longrightarrow \mathrm{DC} 2$ \\
\hline
\end{tabular}


Table 6: Continued.

\begin{tabular}{|c|c|c|c|c|}
\hline Facility & Vehicle & $\begin{array}{l}\text { Departure } \\
\text { time }\end{array}$ & $\begin{array}{c}\text { Arriving } \\
\text { time }\end{array}$ & Route \\
\hline \multirow{6}{*}{ DC3 } & V12 & 121 & 1002 & $\mathrm{DC} 3 \longrightarrow \mathrm{C} 83 \longrightarrow \mathrm{C} 98 \longrightarrow \mathrm{C} 95 \longrightarrow \mathrm{C} 77 \longrightarrow \mathrm{C} 73 \longrightarrow \mathrm{C} 93 \longrightarrow \mathrm{C} 75 \longrightarrow \mathrm{DC} 3$ \\
\hline & V13 & 92 & 831 & $\mathrm{DC} 3 \longrightarrow \mathrm{C} 79 \longrightarrow \mathrm{C} 89 \longrightarrow \mathrm{C} 92 \longrightarrow \mathrm{C} 69 \longrightarrow \mathrm{C} 101 \longrightarrow \mathrm{C} 90 \longrightarrow \mathrm{C} 68 \longrightarrow \mathrm{DC} 3$ \\
\hline & V14 & 109 & 1176 & $\mathrm{DC} 3 \longrightarrow \mathrm{C} 86 \longrightarrow \mathrm{C} 99 \longrightarrow \mathrm{C} 78 \longrightarrow \mathrm{C} 82 \longrightarrow \mathrm{C} 100 \longrightarrow \mathrm{C} 64 \longrightarrow \mathrm{DC} 3$ \\
\hline & V15 & 0 & 865 & $\mathrm{DC} 3 \longrightarrow \mathrm{C} 102 \longrightarrow \mathrm{C} 81 \longrightarrow \mathrm{C} 85 \longrightarrow \mathrm{C} 76 \longrightarrow \mathrm{C} 70 \longrightarrow \mathrm{C} 80 \longrightarrow \mathrm{C} 97 \longrightarrow \mathrm{C} 88 \longrightarrow \mathrm{DC} 3$ \\
\hline & V16 & 0 & 936 & $\mathrm{DC} 3 \longrightarrow \mathrm{C} 74 \longrightarrow \mathrm{C} 67 \longrightarrow \mathrm{C} 84 \longrightarrow \mathrm{C} 87 \longrightarrow \mathrm{C} 71 \longrightarrow \mathrm{C} 72 \longrightarrow \mathrm{C} 91 \longrightarrow \mathrm{C} 65 \longrightarrow \mathrm{C} 96 \longrightarrow \mathrm{DC} 3$ \\
\hline & V17 & 0 & 295 & $\mathrm{DC} 3 \longrightarrow \mathrm{C} 94 \longrightarrow \mathrm{C} 66 \longrightarrow \mathrm{DC} 3$ \\
\hline \multirow{6}{*}{ PC1 } & V18 & 0 & 353 & $\mathrm{PC} 1 \longrightarrow \mathrm{C} 114 \longrightarrow \mathrm{C} 145 \longrightarrow \mathrm{C} 119 \longrightarrow \mathrm{PC} 1$ \\
\hline & V19 & 19 & 891 & $\mathrm{PC} 1 \longrightarrow \mathrm{C} 117 \longrightarrow \mathrm{C} 125 \longrightarrow \mathrm{C} 121 \longrightarrow \mathrm{C} 120 \longrightarrow \mathrm{C} 130 \longrightarrow \mathrm{C} 113 \longrightarrow \mathrm{C} 128 \longrightarrow \mathrm{C} 111 \longrightarrow \mathrm{C} 118 \longrightarrow \mathrm{PC} 1$ \\
\hline & V20 & 4 & 992 & $\mathrm{PC} 1 \longrightarrow \mathrm{C} 115 \longrightarrow \mathrm{C} 144 \longrightarrow \mathrm{C} 116 \longrightarrow \mathrm{C} 123 \longrightarrow \mathrm{C} 140 \longrightarrow \mathrm{C} 122 \longrightarrow \mathrm{C} 106 \longrightarrow \mathrm{C} 139 \longrightarrow \mathrm{C} 124 \longrightarrow \mathrm{PC} 1$ \\
\hline & $\mathrm{V} 21$ & 95 & 1103 & $\mathrm{PC} 1 \longrightarrow \mathrm{C} 135 \longrightarrow \mathrm{C} 104 \longrightarrow \mathrm{C} 136 \longrightarrow \mathrm{C} 103 \longrightarrow \mathrm{C} 147 \longrightarrow \mathrm{C} 142 \longrightarrow \mathrm{C} 141 \longrightarrow \mathrm{C} 143 \longrightarrow \mathrm{C} 112 \longrightarrow \mathrm{PC} 1$ \\
\hline & $\mathrm{V} 22$ & 138 & 951 & $\mathrm{PC} 1 \longrightarrow \mathrm{C} 148 \longrightarrow \mathrm{C} 133 \longrightarrow \mathrm{C} 146 \longrightarrow \mathrm{C} 107 \longrightarrow \mathrm{C} 131 \longrightarrow \mathrm{C} 137 \longrightarrow \mathrm{C} 138 \longrightarrow \mathrm{PC} 1$ \\
\hline & V23 & 0 & 1055 & $\mathrm{PC} 1 \longrightarrow \mathrm{C} 134 \longrightarrow \mathrm{C} 132 \longrightarrow \mathrm{C} 105 \longrightarrow \mathrm{C} 127 \longrightarrow \mathrm{C} 129 \longrightarrow \mathrm{C} 126 \longrightarrow \mathrm{C} 110 \longrightarrow \mathrm{C} 109 \longrightarrow \mathrm{C} 108 \longrightarrow \mathrm{PC} 1$ \\
\hline \multirow{4}{*}{ PC2 } & $\mathrm{V} 24$ & 0 & 883 & $\mathrm{PC} 2 \longrightarrow \mathrm{C} 165 \longrightarrow \mathrm{C} 162 \longrightarrow \mathrm{C} 153 \longrightarrow \mathrm{C} 151 \longrightarrow \mathrm{C} 170 \longrightarrow \mathrm{C} 168 \longrightarrow \mathrm{C} 158 \longrightarrow \mathrm{PC} 2$ \\
\hline & V25 & 37 & 1067 & $\mathrm{PC} 2 \longrightarrow \mathrm{C} 149 \longrightarrow \mathrm{C} 179 \longrightarrow \mathrm{C} 174 \longrightarrow \mathrm{C} 176 \longrightarrow \mathrm{C} 169 \longrightarrow \mathrm{C} 166 \longrightarrow \mathrm{C} 159 \longrightarrow 0$ \\
\hline & V26 & 0 & 1115 & $\mathrm{PC} 2 \longrightarrow \mathrm{C} 156 \longrightarrow \mathrm{C} 172 \longrightarrow \mathrm{C} 167 \longrightarrow \mathrm{C} 157 \longrightarrow \mathrm{C} 155 \longrightarrow \mathrm{C} 152 \longrightarrow \mathrm{C} 163 \longrightarrow \mathrm{C} 150 \longrightarrow \mathrm{PC} 2$ \\
\hline & V27 & 18 & 819 & $\mathrm{PC} 2 \longrightarrow \mathrm{C} 171 \longrightarrow \mathrm{C} 178 \longrightarrow \mathrm{C} 160 \longrightarrow \mathrm{C} 161 \longrightarrow \mathrm{C} 164 \longrightarrow \mathrm{C} 177 \longrightarrow \mathrm{C} 154 \longrightarrow \mathrm{C} 173 \longrightarrow \mathrm{C} 175 \longrightarrow \mathrm{PC} 2$ \\
\hline \multirow{6}{*}{ PC3 } & V28 & 202 & 1031 & $\mathrm{PC} 3 \longrightarrow \mathrm{C} 192 \longrightarrow \mathrm{C} 201 \longrightarrow \mathrm{C} 182 \longrightarrow \mathrm{C} 184 \longrightarrow \mathrm{PC} 3$ \\
\hline & V29 & 127 & 918 & $\mathrm{PC} 3 \longrightarrow \mathrm{C} 220 \longrightarrow \mathrm{C} 183 \longrightarrow \mathrm{C} 193 \longrightarrow \mathrm{C} 214 \longrightarrow \mathrm{C} 207 \longrightarrow \mathrm{C} 217 \longrightarrow \mathrm{C} 205 \longrightarrow \mathrm{C} 202 \longrightarrow \mathrm{C} 180 \longrightarrow \mathrm{PC} 3$ \\
\hline & V30 & 0 & 720 & $\mathrm{PC} 3 \longrightarrow \mathrm{C} 206 \longrightarrow \mathrm{C} 211 \longrightarrow \mathrm{C} 209 \longrightarrow \mathrm{C} 212 \longrightarrow \mathrm{C} 200 \longrightarrow \mathrm{C} 215 \longrightarrow \mathrm{C} 196 \longrightarrow \mathrm{PC} 3$ \\
\hline & V31 & 23 & 1121 & $\mathrm{PC} 3 \longrightarrow \mathrm{C} 191 \longrightarrow \mathrm{C} 185 \longrightarrow \mathrm{C} 187 \longrightarrow \mathrm{C} 188 \longrightarrow \mathrm{C} 216 \longrightarrow \mathrm{C} 199 \longrightarrow \mathrm{PC} 3$ \\
\hline & V32 & 217 & 674 & $\mathrm{PC} 3 \longrightarrow \mathrm{C} 181 \longrightarrow \mathrm{C} 213 \longrightarrow \mathrm{C} 219 \longrightarrow \mathrm{C} 203 \longrightarrow \mathrm{C} 195 \longrightarrow \mathrm{C} 210 \longrightarrow \mathrm{PC} 3$ \\
\hline & V33 & 66 & 918 & $\mathrm{PC} 3 \longrightarrow \mathrm{C} 204 \longrightarrow \mathrm{C} 198 \longrightarrow \mathrm{C} 194 \longrightarrow \mathrm{C} 197 \longrightarrow \mathrm{C} 186 \longrightarrow \mathrm{C} 208 \longrightarrow \mathrm{C} 218 \longrightarrow \mathrm{C} 190 \longrightarrow \mathrm{C} 189 \longrightarrow \mathrm{PC} 3$ \\
\hline
\end{tabular}

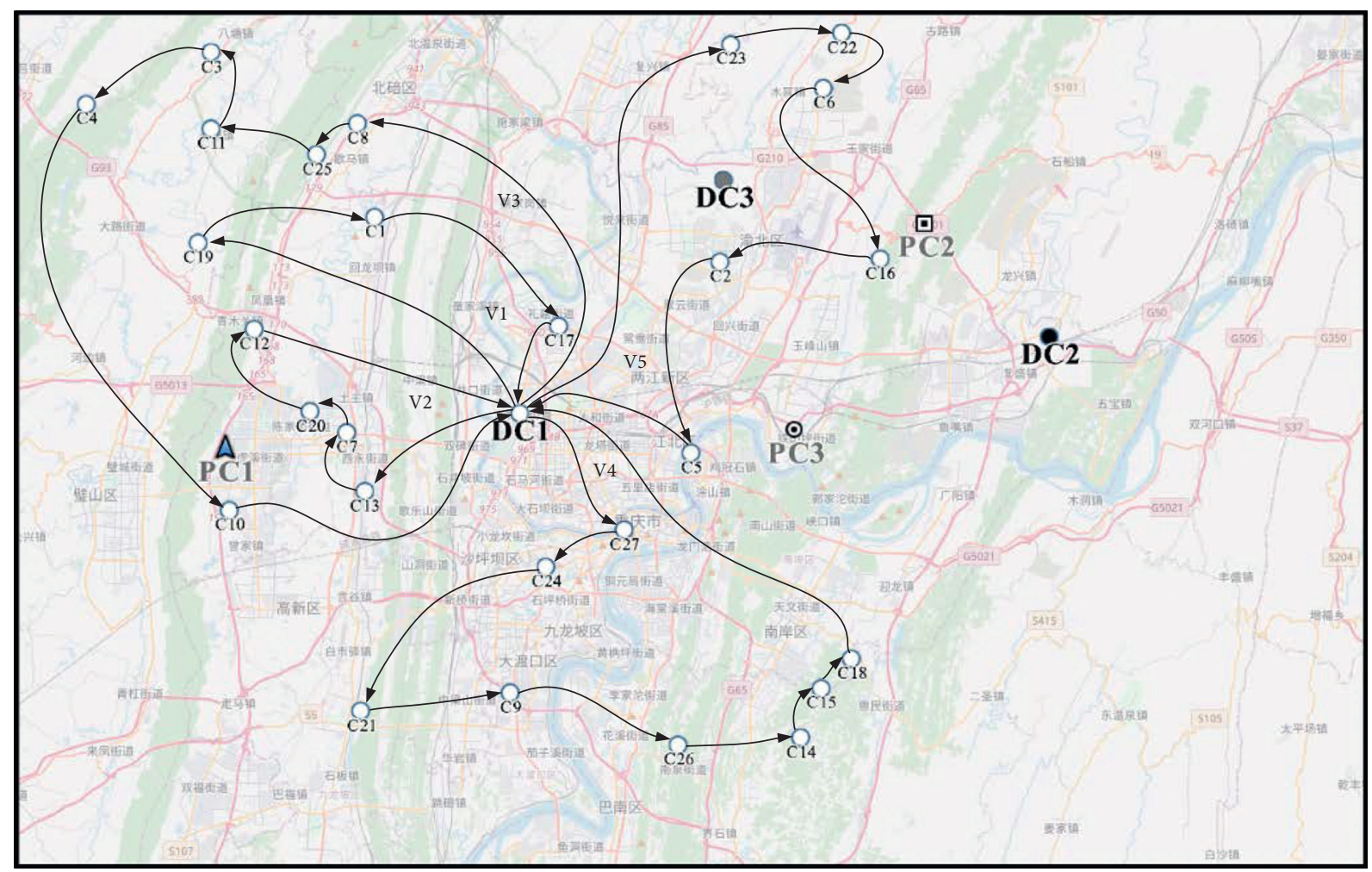

FIgURE 4: Initial vehicle service routes of DC1.

In Figure 7, the initial TTC of DC1, DC2, DC3, PC1, PC2, and PC3 is \$2210, \$3581, \$3730, \$4900, \$3067, and \$3506, respectively. The optimized TTC of each facility is $\$ 2047$, \$1738, \$1134, \$2064, \$2025, and \$2361, respectively. The difference of TTC before and after optimization is relatively obvious. Although the transportation cost generated by the centralized transportation is $\$ 1651$, the logistics network is still significantly optimized on the whole. 
TABLE 7: Relevant parameter setting.

\begin{tabular}{lccc}
\hline Parameter & Numerical value & Parameter & Numerical value \\
\hline$P_{E}$ & $\$ 35$ per hour & $C_{b}$ & 1000 \\
$P_{L}$ & $\$ 35$ per hour & $C_{f}$ & 2000 \\
$W$ & 52 & $M_{v}$ & $\$ 13000$ \\
$T$ & 7 & $M_{b}$ & $\$ 20000$ \\
$T_{v}$ & 10 hour & {$\left[I_{D C 1}, I_{D C 2}, I_{D C 3}, I_{P C 1}, I_{P C 2}, I_{P C 3}\right]$} & {$[\$ 315, \$ 427, \$ 533, \$ 578, \$ 612, \$ 590]$} \\
$T_{b}$ & 15 hour & Population size & 300 \\
$f_{v}$ & 0.363 gallon per miles & Maximum generations & 1000 \\
$f_{b}$ & 0.566 gallon per miles & Crossover probability & 0.8 \\
$P_{v}$ & $\$ 6.18$ per gallon & Mutation probability & 0.2 \\
$P_{b}$ & $\$ 6.18$ per gallon & Travel speed of vehicle & 40 \\
$C_{v}$ & 200 & Travel speed of truck & 60 \\
\hline
\end{tabular}

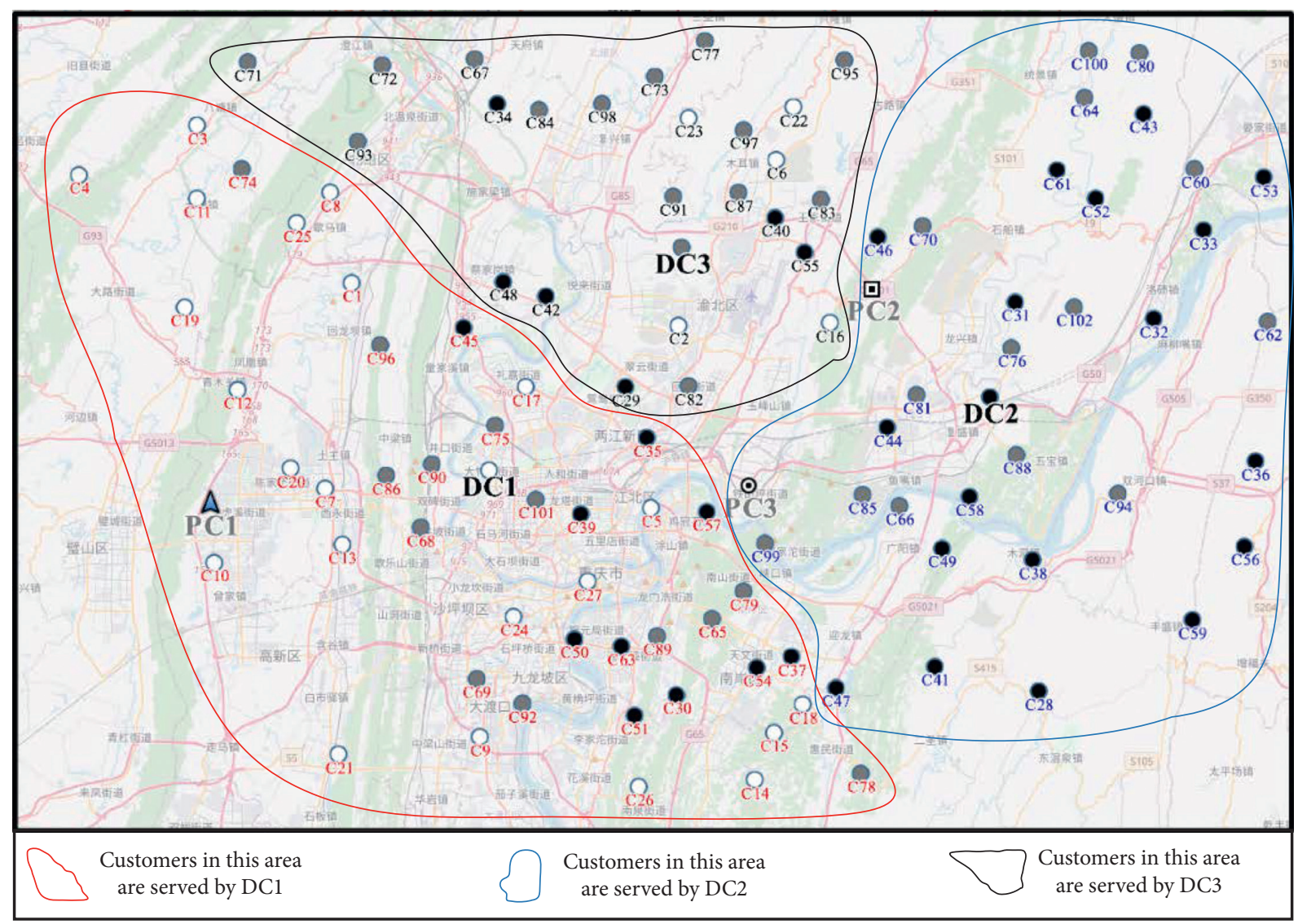

(a)

Figure 5: Continued. 


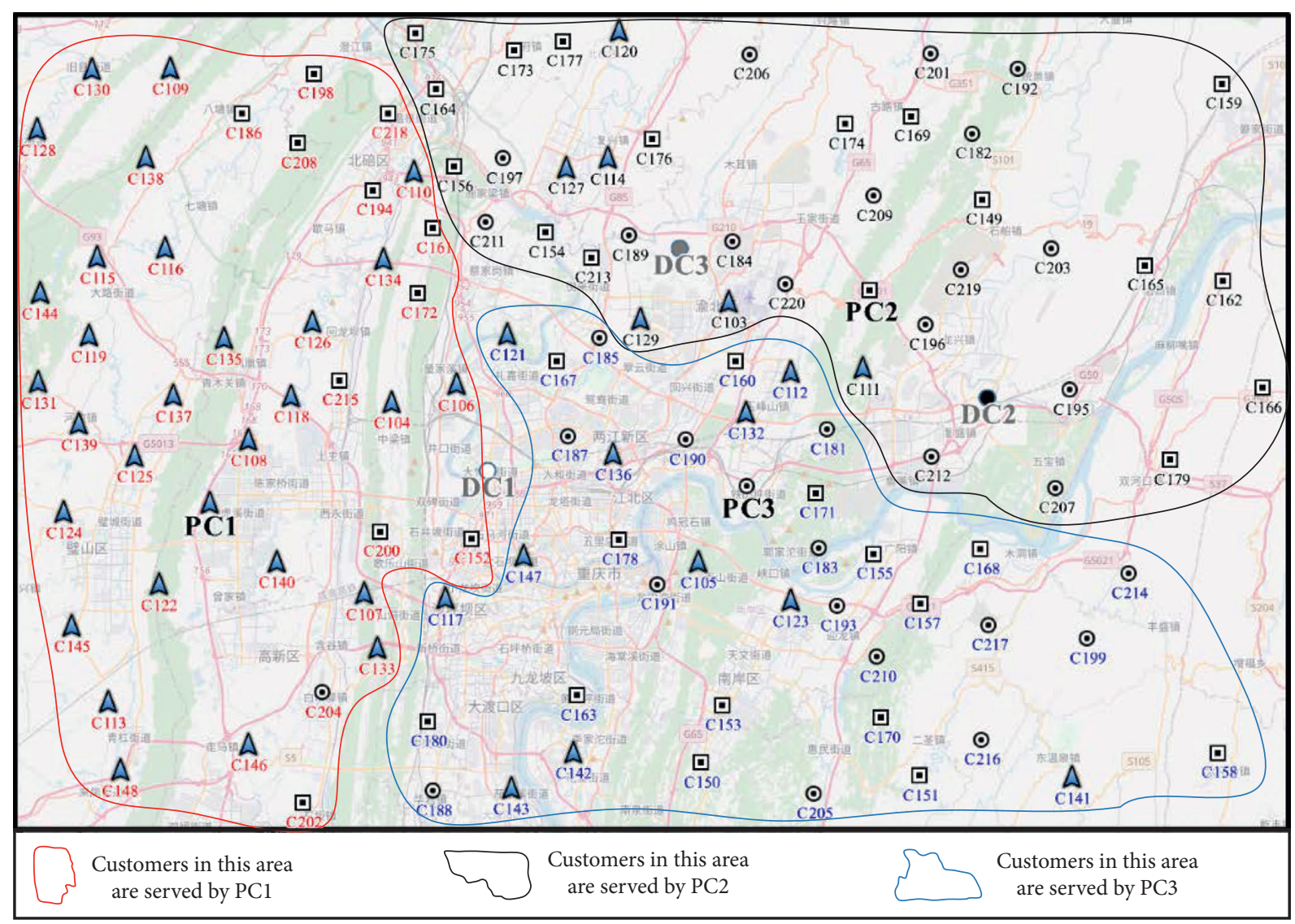

(b)

Figure 5: Customer clustering results with k-means algorithm. (a) Customer clustering results with DCs as cluster centers. (b) Customer clustering results with PCs as cluster centers.
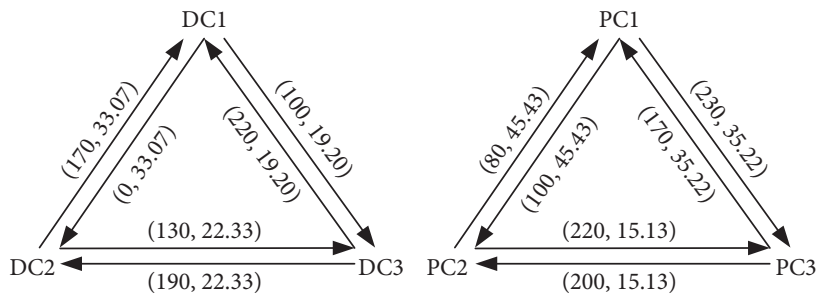

$(*, \#): *$ is the number of transported goods, \# is the distance between facilities

Figure 6: Description of transmissions and distances among facilities.

TABLE 8: Optimized truck routes of centralized transportation.

\begin{tabular}{lc}
\hline Truck & Service routes \\
\hline & $\mathrm{DC} 1 \longrightarrow \mathrm{DC} 2 \longrightarrow \mathrm{DC} 1$ \\
$\mathrm{~T} 1$ & $\mathrm{DC} 2 \longrightarrow \mathrm{DC} 3 \longrightarrow \mathrm{DC} 1 \longrightarrow \mathrm{DC} 2$ \\
& $\mathrm{DC} 3 \longrightarrow \mathrm{DC} 2 \longrightarrow \mathrm{DC} 1 \longrightarrow \mathrm{DC} 3$ \\
\hline & $\mathrm{PC} 1 \longrightarrow \mathrm{PC} 2 \longrightarrow \mathrm{PC} 3 \longrightarrow \mathrm{PC} 1$ \\
$\mathrm{~T} 2$ & $\mathrm{PC} 2 \longrightarrow \mathrm{PC} 3 \longrightarrow \mathrm{PC} 1 \longrightarrow \mathrm{PC} 2$ \\
& $\mathrm{PC} 3 \longrightarrow \mathrm{PC} 2>\mathrm{PC} 1 \longrightarrow \mathrm{PC} 3$ \\
\hline
\end{tabular}

6.4. Analysis and Discussion. The optimization of the logistics network is divided into four cases based on the RS strategy to analyze the influence of RS on the optimization of the logistics network. In Case 1, customer information and transportation resources are privately owned by each logistics facility. In Case 2, RS is adopted by DCs (i.e., DC1, DC2, and DC3). Correspondingly, in Case 3, the members participating in RS are PCs (i.e., PC1, PC2, and PC3). In Case 4, all six facilities receive the RS strategy. Transportation resources can be used jointly by DCs and PCs in Case 4. Table 11 and Figure 8 show the numerical optimization results of the logistics network in the four scenarios.

In Table 11 and Figure 8, Case 4 outperforms Cases 2 and 3 in most aspects. First, the TOC of Cases 2 and 3 is $\$ 17038$ and $\$ 16977$, respectively, which are larger than 


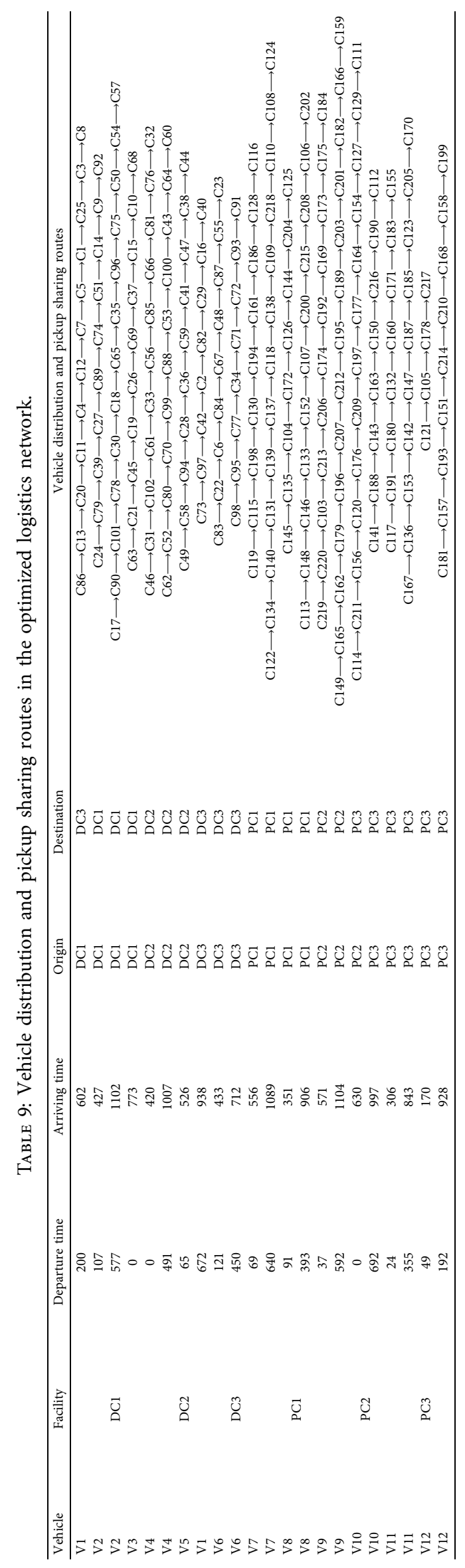


TABle 10: Comparison before and after optimization.

\begin{tabular}{|c|c|c|c|c|c|c|c|c|c|c|c|c|}
\hline \multirow{3}{*}{ Facility } & \multicolumn{4}{|c|}{ Initial } & \multicolumn{5}{|c|}{ Optimized } & \multicolumn{3}{|c|}{ Gap } \\
\hline & \multicolumn{4}{|c|}{ TOC } & \multirow[t]{2}{*}{$\mathrm{NV}$} & \multicolumn{4}{|c|}{ TOC } & \multirow{2}{*}{ NV } & \multirow{2}{*}{ TOC } & \multirow{2}{*}{$\mathrm{NV}$} \\
\hline & TTC & TFC & TPC & TMC & & TTC & TFC & TPC & TMC & & & \\
\hline $\mathrm{DC1}$ & 2210 & 315 & 27 & 179 & 5 & 2047 & 315 & 0 & \multirow{6}{*}{429} & \multirow{6}{*}{12} & \multirow{6}{*}{8859} & \multirow{6}{*}{19} \\
\hline DC2 & 3581 & 427 & 55 & 214 & 6 & 1738 & 427 & 0 & & & & \\
\hline DC3 & 3730 & 533 & 44 & 214 & 6 & 1134 & 533 & 0 & & & & \\
\hline PC1 & 4900 & 578 & 65 & 214 & 6 & 2064 & 578 & 0 & & & & \\
\hline PC2 & 3067 & 612 & 22 & 143 & 4 & 2025 & 612 & 0 & & & & \\
\hline PC3 & 3506 & 590 & 33 & 214 & 6 & 2361 & 590 & 0 & & & & \\
\hline Centralized transportation & - & - & - & - & - & 1651 & - & 0 & 110 & 2 & & \\
\hline Total & \multicolumn{4}{|c|}{25473} & 33 & \multicolumn{4}{|c|}{16614} & 14 & - & - \\
\hline
\end{tabular}

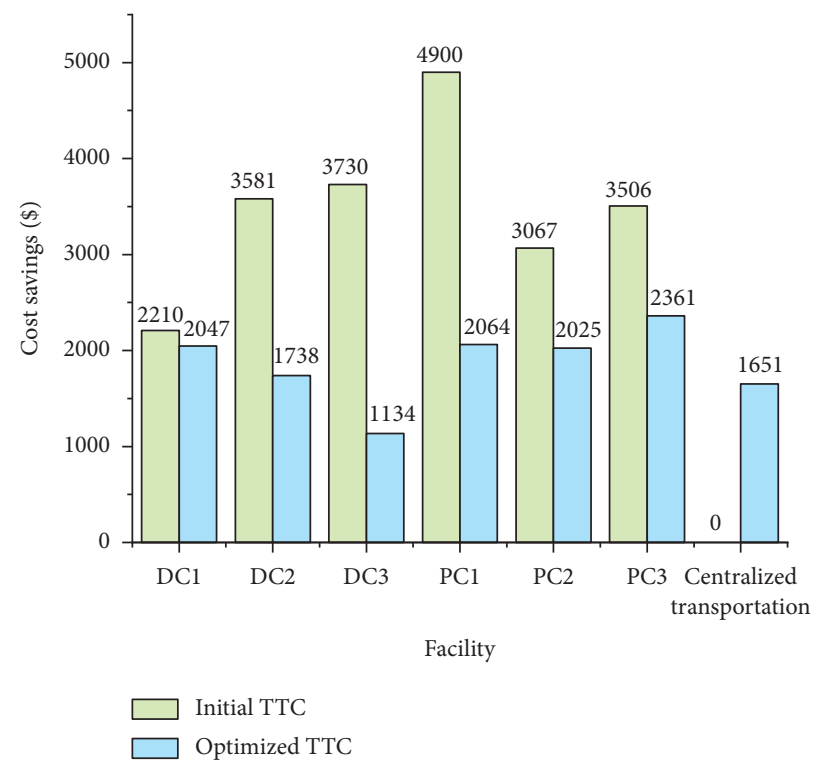

Figure 7: Comparison results of TTC before and after optimization.

TABle 11: Comparison results of the four cases.

\begin{tabular}{lcccccccr}
\hline Cases & TTC $(\$)$ & TPC $(\$)$ & TFC $(\$)$ & NV & TMC $(\$)$ & TOC $(\$)$ & NV & TTC (\$) \\
\hline Case 1 & 20994 & 246 & 3055 & 33 & 1178 & 25473 & & \\
Case 2 & 17038 & 120 & 3055 & 23 & 841 & 21053 & 10 & 3956 \\
Case 3 & 16977 & 126 & 3055 & 24 & 876 & 21034 & 9 & 4017 \\
Case 4 & 13020 & 0 & 3055 & 14 & 539 & 16614 & 19 & 7974 \\
\hline
\end{tabular}

$\$ 13020$ in Case 4. Second, the number of vehicles and the TMCs of Scenario 4 is 14 and $\$ 539$, respectively, which indicates that Case 4 is more conducive to saving resources. Finally, the gap of TOC between Cases 4 and 1 is $\$ 8859$. Therefore, if the six logistics facilities adopt RS simultaneously, then the logistics costs can be optimized better and the transportation resources can be saved considerably.

6.5. Management Insights. In this study, the RS strategy optimizes the logistics network significantly by reallocating customers, including the logistics operation costs and the number of vehicles. Therefore, the management insights obtained in this study are as follows:

(1) In a multi-depot, large-scale logistics network with pickups and deliveries, customer information, facility capacity, and transportation resources can be shared to amplify resource utilization by introducing RS strategies. RS is not only conducive to the operation of logistics facilities, including the use of logistics costs and transport resources, but also conducive for providing customers with more convenient logistics services. In a logistics network with vehicle sharing, vehicles are used multiple times within and between facilities to avoid idle 


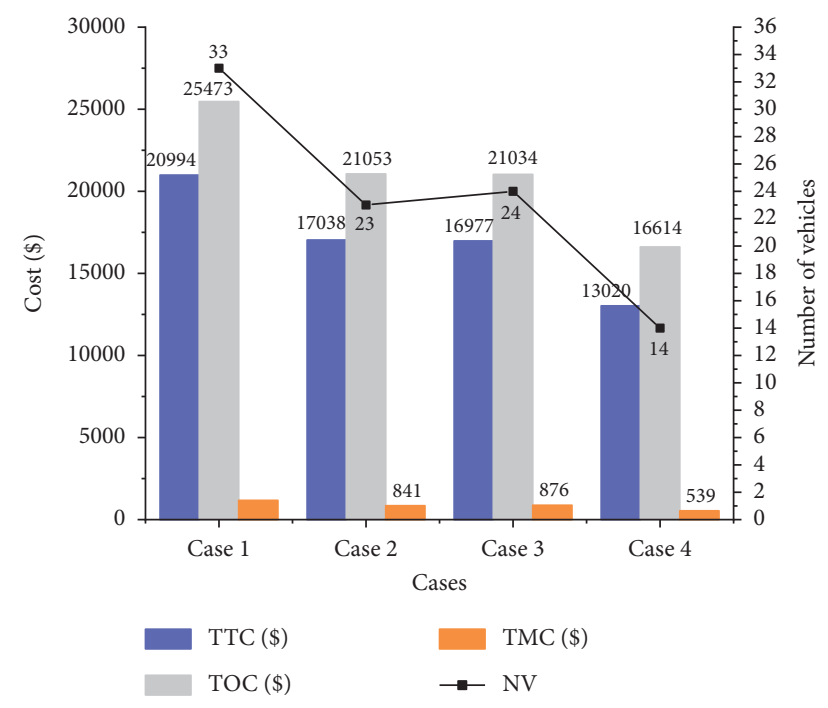

Figure 8: Comparison results of TTC, TC, TMC, and NV in the four cases.

vehicle resources. In addition, the sharing of customer information helps facilities provide logistics services to customers with higher quality. Therefore, participating actively in RS is remarkably necessary for LSPs.

(2) From the perspective of local traffic management departments and environmental departments, the optimization of the logistics network through RS not only reduces transportation resources but also relieves the local traffic pressure. In addition, the efficient use of resources promotes the development of green environment. Therefore, local government departments can actively support and give incentive policies to promote RS among local logistics facilities to optimize the logistics network and ease the traffic environment. Moreover, the effective sharing mechanism in the multicenter logistics network with pickups and deliveries can enhance the efficient operation of the logistics system and promote the green and sustainable development of the local intelligent logistics system. Therefore, the introduction of RS can promote the construction and development of local smart and green urban logistics with the incentive of departments and the active participation of facilities.

\section{Conclusions}

This study handles the MDPDPRS, which optimizes the logistics network by sharing customer and transportation resources. The reconfiguration of resources and customers improve the operating efficiency of the logistics network. The main contributions of this study include the following aspects. First, the MDPDPRS is modeled as a bi-objective mathematical model to optimize the total logistics operating cost and number of vehicles. Second, a two-stage hybrid algorithm is designed to solve the MDPDPRS, which contains the $k$-means, CW, and NSGA-II algorithms. Third, the application of the proposed mathematical model and methodology are improved by the numerical result of a realworld case study and benchmark.

In view of the shared transportation resources and customers' information, a two-stage algorithm is designed, which initially clusters customers to reconfigure the resources and then optimizes the vehicle routes. On the one hand, $k$-means algorithm, which clusters customers based on Manhattan distance, helps reduce the difficulty of solving MDPDPRS and enables vehicle resources to be used in a centralized manner multiple times. On the other hand, the combination of CW and NSGA-II algorithms improves the global searching capability and the speed of the algorithm in finding the Pareto optimal solutions.

The numerical results of a real-word case study, which is obtained in Chongqing, China, is discussed and analyzed to improve the application and performance of the designed mathematical model and methodology in solving practical problems similar to the MDPDPRS. The gap of the logistics operating costs and number of vehicles before and after the optimization are $\$ 8859$ and 19 , respectively, which verify the effectiveness of the model and methodology proposed in this study. In addition, the algorithm comparison results of benchmarks (from C-mdvrptw datasets) verify that the proposed KCW-NSGA-II algorithm is superior to the standard NSGA-II, GA-PSO, and MOPSO. The results of numerical discussion on the four cases in which different RS strategies are adopted prove that RS is helpful to optimize logistics costs and save transportation resources.

In this study, the bi-objective mathematical model and methodology of MDPDPRS are designed, which provide references for the reconfiguration of resources and the optimization of logistics operation costs. In view of the limitations of the current study and the dynamic development of the logistics industry, further research can be considered from the following aspects. (1) The dynamic change in customer demands and customer satisfaction are the two aspects that the realistic LSPs focus on, and these aspects can be added into the study of MDPDPRS. (2) Constructing a dynamic mathematical programming model and designing an exact algorithm to find the exact solution of MDPDPRS are worthy of research. (3) Exploring the approaches to realize RS in a large logistics network and the means to reduce the effect of logistics transportation on the environment can be considered in the study of MDPDPRS. (4) Considering the cost sharing mechanism under the RS mechanism to promote the formation of collaboration and maintain its stability can enrich the study of MDPDPRS.

\section{Data Availability}

The data used to support the findings of this study are included within the article.

\section{Conflicts of Interest}

The authors declare that they do not have any conflicts of interest. 


\section{Acknowledgments}

This research was supported by National Natural Science Foundation of China (Project Nos. 71871035 and 41977337), the Fundamental Research Funds for the Central Universities (grant no. 22120210009), Humanity and Social Science Youth Foundation of Ministry of Education of China (18YJC630189), Key Science and Technology Research Project of Chongqing Municipal Education Commission (KJZD-K202000702), Key Project of Human Social Science of Chongqing Municipal Education Commission (No. 20SKGH079), Social Science Foundation of Chongqing of China (2019YBGL054), and Chongqing Graduate Tutor Team Construction Project (No. JDDSTD2019008). This research was also supported by 2018 Chongqing Liuchuang Plan Innovation Project (cx2018111).

\section{References}

[1] C. K. M. Lee, Y. Lv, K. K. H. Ng, W. Ho, and K. L. Choy, "Design and application of Internet of things-based warehouse management system for smart logistics," International Journal of Production Research, vol. 56, no. 8, pp. 2753-2768, 2017.

[2] S. Liu, Y. Zhang, Y. Liu, L. Wang, and X. V. Wang, "An "Internet of Things" enabled dynamic optimization method for smart vehicles and logistics tasks," Journal of Cleaner Production, vol. 215, pp. 806-820, 2019.

[3] S. A. R. Khan, C. Jian, Y. Zhang, H. Golpîra, A. Kumar, and A. Sharif, "Environmental, social and economic growth indicators spur logistics performance: from the perspective of South Asian Association for Regional Cooperation countries," Journal of Cleaner Production, vol. 214, pp. 1011-1023, 2019.

[4] O. Seroka-Stolka, "The development of green logistics for implementation sustainable development strategy in companies," Procedia - Social and Behavioral Sciences, vol. 151, pp. 302-309, 2014.

[5] G. Ann E, K. Uday S, and S. Abraham, "Design and operation of an order-consolidation warehouse: models and application," European Journal of Operational Research, vol. 58, no. 1, pp. 14-36, 1992.

[6] T. C. Poon, K. L. Choy, H. K. H. Chow, H. C. W. Lau, F. T. S. Chan, and K. C. Ho, "A RFID case-based logistics resource management system for managing order-picking operations in warehouses," Expert Systems with Applications, vol. 36, no. 4, pp. 8277-8301, 2009.

[7] R. Dekker, J. Bloemhof, and I. Mallidis, "Operations Research for green logistics - an overview of aspects, issues, contributions and challenges," European Journal of Operational Research, vol. 219, no. 3, pp. 671-679, 2012.

[8] Y. Yu, C. Yu, G. Xu, R. Y. Zhong, and G. Q. Huang, “An operation synchronization model for distribution center in E-commerce logistics service," Advanced Engineering Informatics, vol. 43, 2020.

[9] S. A. R. Khan, "The nexus between carbon emissions, poverty, economic growth, and logistics operations-empirical evidence from southeast Asian countries," Environmental Science and Pollution Research, vol. 26, no. 13, pp. 13210-13220, 2019.

[10] W. Zhang, M. Zhang, W. Zhang, Q. Zhou, and X. Zhang, "What influences the effectiveness of green logistics policies? A grounded theory analysis," Science of the Total Environment, vol. 714, Article ID 136731, 2020.
[11] M. Gansterer and R. F. Hartl, "Shared resources in collaborative vehicle routing," Top, vol. 28, no. 1, pp. 1-20, 2020.

[12] M. Mirabi, S. M. T. Fatemi Ghomi, and F. Jolai, "Efficient stochastic hybrid heuristics for the multi-depot vehicle routing problem," Robotics and Computer-Integrated Manufacturing, vol. 26, no. 6, pp. 564-569, 2010.

[13] M. D. A. Serna, C. A. S. Uran, J. A. Z. Cortes, and A. F. A. Benitez, "Vehicle routing to multiple warehouses using a memetic algorithm," Procedia - Social and Behavioral Sciences, vol. 160, pp. 587-596, 2014.

[14] Y. Wang, J. Zhang, K. Assogba, Y. Liu, M. Xu, and Y. Wang, "Collaboration and transportation resource sharing in multiple centers vehicle routing optimization with delivery and pickup," Knowledge-Based Systems, vol. 160, pp. 296-310, 2018.

[15] M. Zhang, S. Pratap, G. Q. Huang, and Z. Zhao, "Optimal collaborative transportation service trading in $\mathrm{B} 2 \mathrm{~B}$ e-commerce logistics," International Journal of Production Research, vol. 55, no. 18, pp. 5485-5501, 2017.

[16] W. Ho, G. T. S. Ho, P. Ji, and H. C. W. Lau, "A hybrid genetic algorithm for the multi-depot vehicle routing problem," Engineering Applications of Artificial Intelligence, vol. 21, no. 4, pp. 548-557, 2008.

[17] Y. Wang, X. Ma, Z. Li, Y. Liu, M. Xu, and Y. Wang, "Profit distribution in collaborative multiple centers vehicle routing problem," Journal of Cleaner Production, vol. 144, pp. 203-219, 2017.

[18] D. Chen and Z. Yang, "Multiple depots vehicle routing problem in the context of total urban traffic equilibrium," Journal of Advanced Transportation, vol. 2017, Article ID 8524960, 14 pages, 2017.

[19] I. Gribkovskaia, Ø. Halskau, G. Laporte, and M. Vlček, "General solutions to the single vehicle routing problem with pickups and deliveries," European Journal of Operational Research, vol. 180, no. 2, pp. 568-584, 2007.

[20] J. Luo and M.-R. Chen, "Multi-phase modified shuffled frog leaping algorithm with extremal optimization for the MDVRP and the MDVRPTW," Computers \& Industrial Engineering, vol. 72, pp. 84-97, 2014.

[21] S. Deng, Y. Yuan, Y. Wang, H. Wang, and C. Koll, "Collaborative multicenter logistics delivery network optimization with resource sharing," PLoS One, vol. 15, no. 11, Article ID e0242555, 2020.

[22] A. Angelopoulos, D. Gavalas, C. Konstantopoulos, D. Kypriadis, and G. Pantziou, "Incentivized vehicle relocation in vehicle sharing systems," Transportation Research Part C: Emerging Technologies, vol. 97, pp. 175-193, 2018.

[23] Y. Wang, Y. Yuan, X. Guan et al., "Collaborative two-echelon multicenter vehicle routing optimization based on statespace-time network representation," Journal of Cleaner Production, vol. 258, 2020.

[24] L. Verdonck, A. Caris, K. Ramaekers, and G. K. Janssens, "Collaborative logistics from the perspective of road transportation companies," Transport Reviews, vol. 33, no. 6, pp. 700-719, 2013.

[25] C. Defryn and K. Sörensen, "A fast two-level variable neighborhood search for the clustered vehicle routing problem," Computers \& Operations Research, vol. 83, pp. 78-94, 2017.

[26] R. J. Kuo, C. H. Mei, F. E. Zulvia, and C. Y. Tsai, “An application of a metaheuristic algorithm-based clustering ensemble method to APP customer segmentation," Neurocomputing, vol. 205, pp. 116-129, 2016. 
[27] S. Yang, L. Ning, P. Shang, and L. Tong, "Augmented Lagrangian relaxation approach for logistics vehicle routing problem with mixed backhauls and time windows," Transportation Research Part E: Logistics and Transportation Review, vol. 135, 2020.

[28] N. A. Wassan, G. Nagy, and S. Ahmadi, "A heuristic method for the vehicle routing problem with mixed deliveries and pickups," Journal of Scheduling, vol. 11, no. 2, pp. 149-161, 2008.

[29] N. Wassan and G. Nagy, "Vehicle routing problem with deliveries and pickups: modelling issues and meta-heuristics solution approaches," International Journal of Transportation, vol. 2, no. 1, pp. 95-110, 2014.

[30] R. Liu, X. Xie, V. Augusto, and C. Rodriguez, "Heuristic algorithms for a vehicle routing problem with simultaneous delivery and pickup and time windows in home health care," European Journal of Operational Research, vol. 230, no. 3, pp. 475-486, 2013.

[31] Q. Chen, K. Li, and Z. Liu, "Model and algorithm for an unpaired pickup and delivery vehicle routing problem with split loads," Transportation Research Part E: Logistics and Transportation Review, vol. 69, pp. 218-235, 2014.

[32] S. Vaziri, F. Etebari, and B. Vahdani, "Development and optimization of a horizontal carrier collaboration vehicle routing model with multi-commodity request allocation," Journal of Cleaner Production, vol. 224, pp. 492-505, 2019.

[33] Y. Wang, S. Peng, X. Zhou, M. Mahmoudi, and L. Zhen, "Green logistics location-routing problem with eco-packages," Transportation Research Part E: Logistics and Transportation Review, vol. 143, 2020.

[34] E. Benavent, M. Landete, E. Mota, and G. Tirado, "The multiple vehicle pickup and delivery problem with LIFO constraints," European Journal of Operational Research, vol. 243, no. 3, pp. 752-762, 2015.

[35] Y. Wang, Q. Li, X. Guan, M. Xu, Y. Liu, and H. Wang, "Twoechelon collaborative multi-depot multi-period vehicle routing problem," Expert Systems with Applications, vol. 167, 2021.

[36] Y. Wang, S. Peng, C. Xu et al., "Two-echelon logistics delivery and pickup network optimization based on integrated cooperation and transportation fleet sharing," Expert Systems with Applications, vol. 113, pp. 44-65, 2018.

[37] T. Pichpibul and R. Kawtummachai, "An improved Clarke and Wright savings algorithm for the capacitated vehicle routing problem," ScienceAsia, vol. 38, no. 3, 2012.

[38] A. Martínez-Puras and J. Pacheco, "MOAMP-Tabu search and NSGA-II for a real Bi-objective scheduling-routing problem," Knowledge-Based Systems, vol. 112, pp. 92-104, 2016.

[39] J. Luo and M.-R. Chen, "Improved Shuffled Frog Leaping Algorithm and its multi-phase model for multi-depot vehicle routing problem," Expert Systems with Applications, vol. 41, no. 5, pp. 2535-2545, 2014.

[40] S. N. Parragh, K. F. Doerner, and R. F. Hartl, "A survey on pickup and delivery problems," Journal für Betriebswirtschaft, vol. 58, no. 2, pp. 81-117, 2008.

[41] C. Ting and C. H. Chen, "Combination of multiple ant colony system and simulated annealing for the multidepot vehiclerouting problem with time windows transportation research record," Journal of the Transportation Research Board, vol. 2089, no. 1, pp. 85-92, 2008.

[42] S. Yanik, B. Bozkaya, and R. deKervenoael, "A new VRPPD model and a hybrid heuristic solution approach for e-tailing,"
European Journal of Operational Research, vol. 236, no. 3, pp. 879-890, 2014.

[43] M. Alzaqebah, S. Jawarneh, S. Jawarneh, H. M. Sarim, and S. Abdullah, "Bees algorithm for vehicle routing problems with time windows," International Journal of Machine Learning and Computing, vol. 8, no. 3, pp. 236-240, 2018.

[44] M. Oberscheider, J. Zazgornik, M. Gronalt, and P. Hirsch, "An exact approach to minimize the greenhouse gas emissions in timber transport," Journal of Applied Operational Research, vol. 7, no. 2, pp. 43-59, 2015.

[45] M. Oberscheider, J. Zazgornik, C. B. Henriksen, M. Gronalt, and P. Hirsch, "Minimizing driving times and greenhouse gas emissions in timber transport with a near-exact solution approach," Scandinavian Journal of Forest Research, vol. 28, no. 5, pp. 493-506, 2013.

[46] A. Bettinelli, A. Ceselli, and G. Righini, “A branch-and-price algorithm for the multi-depot heterogeneous-fleet pickup and delivery problem with soft time windows," Mathematical Programming Computation, vol. 6, no. 2, pp. 171-197, 2014.

[47] S. Irnich, "A multi-depot pickup and delivery problem with a single hub and heterogeneous vehicles," European Journal of Operational Research, vol. 122, no. 2, pp. 310-328, 2000.

[48] E. Queiroga, Y. Frota, R. Sadykov, A. Subramanian, E. Uchoa, and T. Vidal, "On the exact solution of vehicle routing problems with backhauls," European Journal of Operational Research, vol. 287, no. 1, pp. 76-89, 2020.

[49] T. Leelertkij, P. Parthanadee, J. Buddhakulsomsiri, and A. Lambert, "Vehicle routing problem with transshipment: mathematical model and algorithm," Journal of Advanced Transportation, vol. 2021, Article ID 8886572, 15 pages, 2021.

[50] S. Ropke and J.-F. Cordeau, "Branch and cut and price for the pickup and delivery problem with time windows," Transportation Science, vol. 43, no. 3, pp. 267-286, 2009.

[51] V. Kachitvichyanukul, P. Sombuntham, and S. Kunnapapdeelert, "Two solution representations for solving multi-depot vehicle routing problem with multiple pickup and delivery requests via PSO," Computers \& Industrial Engineering, vol. 89, pp. 125-136, 2015.

[52] A. Soriano, M. Gansterer, and R. F. Hartl, "The two-region multi-depot pickup and delivery problem," OR Spectrum, vol. 40, no. 4, pp. 1077-1108, 2018.

[53] Y. Wang, X. Ma, Y. Lao, and Y. Wang, "A fuzzy-based customer clustering approach with hierarchical structure for logistics network optimization," Expert Systems with Applications, vol. 41, no. 2, pp. 521-534, 2014.

[54] S. Barreto, C. Ferreira, J. Paixão, and B. S. Santos, "Using clustering analysis in a capacitated location-routing problem," European Journal of Operational Research, vol. 179, no. 3, pp. 968-977, 2007.

[55] S. Mitra, "A parallel clustering technique for the vehicle routing problem with split deliveries and pickups," Journal of the Operational Research Society, vol. 59, no. 11, pp. 15321546, 2017.

[56] M. Assari, J. Delaram, and O. Fatahi Valilai, "Mutual manufacturing service selection and routing problem considering customer clustering in Cloud manufacturing," Production \& Manufacturing Research, vol. 6, no. 1, pp. 345-363, 2018.

[57] P. Singanamala, D. Reddy, and P. Venkataramaiah, "Solution to a multi depot vehicle routing problem using K-means algorithm, clarke and wright algorithm and ant colony optimization," International Journal of Applied Engineering Research, vol. 13, no. 21, pp. 15236-15246, 2018. 
[58] R. Nallusamy, K. Duraiswamy, R. Dhanalaksmi, and Parthiban, "Optimization of multiple vehicle routing problems using approximation algorithms," 2010, https://arxiv.org/ftp/ arxiv/papers/1001/1001.4197.pdf.

[59] H. Xu, P. Pu, and F. Duan, "Dynamic vehicle routing problems with enhanced ant colony optimization," Discrete Dynamics in Nature and Society, vol. 2018, Article ID 1295485 , 13 pages, 2018.

[60] M. W. A. Hakim, S. R. Dewi, Y. Windiatmoko, and U. A. Aziz, Optimizing planning service territories by dividing into compact several sub-areas using binary K-means clustering according vehicle constraints, 2010.

[61] S. Mourelo Ferrandez, T. Harbison, T. Weber, R. Sturges, and R. Rich, "Optimization of a truck-drone in tandem delivery network using k-means and genetic algorithm," Journal of Industrial Engineering and Management, vol. 9, no. 2, 2016.

[62] M. Elango, S. Nachiappan, and M. K. Tiwari, "Balancing task allocation in multi-robot systems using $\mathrm{K}$-means clustering and auction based mechanisms," Expert Systems with Applications, vol. 38, no. 6, pp. 6486-6491, 2011.

[63] Y. Li, B. Qian, R. Hu, L.-P. Wu, and B. Liu, "Two-stage algorithm for solving multi-depot green vehicle routing problem with time window," Intelligent Computing Theories and Application, vol. 12, pp. 665-675, 2019.

[64] N. Alikar, S. M. Mousavi, R. A. Raja Ghazilla, M. Tavana, and E. U. Olugu, "Application of the NSGA-II algorithm to a multi-period inventory-redundancy allocation problem in a series-parallel system," Reliability Engineering \& System Safety, vol. 160, pp. 1-10, 2017.

[65] V. Babaveisi, M. M. Paydar, and A. S. Safaei, "Optimizing a multi-product closed-loop supply chain using NSGA-II, MOSA, and MOPSO meta-heuristic algorithms," Journal of Industrial Engineering International, vol. 14, no. 2, pp. 305326, 2017.

[66] O. Kaiwartya, S. Kumar, D. K. Lobiyal, P. K. Tiwari, A. H. Abdullah, and A. N. Hassan, "Multiobjective dynamic vehicle routing problem and time seed based solution using particle swarm optimization," Journal of Sensors, vol. 2015, Article ID 189832, 14 pages, 2015.

[67] Z. Cui, Y. Chang, J. Zhang, X. Cai, and W. Zhang, "Improved NSGA-III with selection-and-elimination operator," Swarm and Evolutionary Computation, vol. 49, pp. 23-33, 2019.

[68] G. Srivastava, A. Singh, and R. Mallipeddi, "NSGA-II with objective-specific variation operators for multiobjective vehicle routing problem with time windows," Expert Systems with Applications, vol. 176, Article ID 114779, 2021.

[69] F. Maadanpour Safari, F. Etebari, and A. Pourghader Chobar, "Modelling and optimization of a tri-objective Transportation-Location-Routing Problem considering route reliability: using MOGWO, MOPSO, MOWCA and NSGA-II," Journal of Optimization in Industrial Engineering, vol. 14, no. 2, pp. 99-114, 2021.

[70] M. Shafiei Nikabadi, E. Molayi, and M. Akhavan Rad, "Optimization of vehicle routing problem under uncertainty with emphasis on green-lean practices and customer satisfaction," Journal of Transportation Research, vol. 18, no. 1, pp. 113-134, 2021.

[71] M. Rabbani, N. Oladzad-Abbasabady, and N. AkbarianSaravi, "Ambulance routing in disaster response considering variable patient condition: NSGA-II and MOPSO algorithms," Journal of Industrial Management Optimization, vol. 13, no. 5, 2017.
[72] M. Nourinejad and M. J. Roorda, "A dynamic carsharing decision support system," Transportation Research Part E: Logistics and Transportation Review, vol. 66, pp. 36-50, 2014.

[73] J. Li, R. Wang, T. Li, Z. Lu, and P. M. Pardalos, "Benefit analysis of shared depot resources for multi-depot vehicle routing problem with fuel consumption," Transportation Research Part D: Transport and Environment, vol. 59, pp. 417-432, 2018.

[74] R. Nair and E. Miller-Hooks, "Fleet management for vehicle sharing operations," Transportation Science, vol. 45, no. 4, pp. 524-540, 2011.

[75] Y. Zou, B. Lin, X. Yang, L. Wu, M. Muneeb Abid, and J. Tang, "Application of the bayesian model averaging in analyzing freeway traffic incident clearance time for emergency management," Journal of Advanced Transportation, vol. 2021, Article ID 6671983, 9 pages, 2021.

[76] T. Pichpibul and R. Kawtummachai, "A heuristic approach based on Clarke-Wright algorithm for open vehicle routing problem," The Scientific World Journal, vol. 2013, Article ID 874349, 11 pages, 2013.

[77] H. Mei, Y. Jingshuai, M. Teng, L. Xiuli, and W. Ting, "The modeling of milk-run vehicle routing problem based on improved C-W algorithm that joined time window," Transportation Research Procedia, vol. 25, pp. 716-728, 2017.

[78] İ. K. Altınel and T. Öncan, "A new enhancement of the Clarke and Wright savings heuristic for the capacitated vehicle routing problem," Journal of the Operational Research Society, vol. 56, no. 8, pp. 954-961, 2017.

[79] B. Vahdani, F. Mansour, M. Soltani, and D. Veysmoradi, "Biobjective optimization for integrating quay crane and internal truck assignment with challenges of trucks sharing," Knowledge-Based Systems, vol. 163, pp. 675-692, 2019. 